\title{
Vibration Performance Analysis of a Mining Vehicle with Bounce and Pitch Tuned Hydraulically Interconnected Suspension
}

Jie Zhang ${ }^{1}$, Yuanwang Deng ${ }^{1 *}$, Nong Zhang ${ }^{2}$, Bangji Zhang ${ }^{1}$, Hengmin Qi ${ }^{1}$ and Minyi Zheng ${ }^{3}$

\begin{abstract}
The current investigations primarily focus on using advanced suspensions to overcome the tradeoff design of ride comfort and handling performance for mining vehicles. It is generally realized by adjusting spring stiffness or damping parameters through active control methods. However, some drawbacks regarding control complexity and uncertain reliability are inevitable for these advanced suspensions. Herein, a novel passive hydraulically interconnected suspension (HIS) system is proposed to achieve an improved ride-handling compromise of mining vehicles. A lumped-mass vehicle model involved with a mechanical-hydraulic coupled system is developed by applying the free-body diagram method. The transfer matrix method is used to derive the impedance of the hydraulic system, and the impedance is integrated to form the equation of motions for a mechanical-hydraulic coupled system. The modal analysis method is employed to obtain the free vibration transmissibilities and force vibration responses under different road excitations. A series of frequency characteristic analyses are presented to evaluate the isolation vibration performance between the mining vehicles with the proposed HIS and the conventional suspension. The analysis results prove that the proposed HIS system can effectively suppress the pitch motion of sprung mass to guarantee the handling performance, and favorably provide soft bounce stiffness to improve the ride comfort. The distribution of dynamic forces between the front and rear wheels is more reasonable, and the vibration decay rate of sprung mass is increased effectively. This research proposes a new suspension design method that can achieve the enhanced cooperative control of bounce and pitch motion modes to improve the ride comfort and handling performance of mining vehicles as an effective passive suspension system.
\end{abstract}

Keywords: Hydraulically interconnected suspension, Transfer matrix method, Modal vibration analysis, Ride comfort, Handling performance, Mining vehicle

\section{Introduction}

Mining vehicles are an important underground device for the transportation of minerals and staff. Recently, higher requirements on their isolation vibration performance have been demanded. However, the vehicle body often experiences continuous and excessive vibrations owing to harsh working conditions. This can lead to discomfort and seriously affect the physical and mental health of staff [1]. Moreover, the long longitudinal distance between the

\footnotetext{
*Correspondence: dengyuanwang610@126.com

1 State Key Laboratory of Advanced Design and Manufacturing for Vehicle Body, College of Mechanical and Vehicle Engineering, Hunan University, Changsha 410082, China

Full list of author information is available at the end of the article
}

axles and large load change in mining vehicles can aggravate the vehicle body pitch motion under continuous upslope and downslope roads in the mines. The severe pitch motion is the primary source of the longitudinal vibration to be experienced at points above the center of gravity (CG) of the vehicle body. Simultaneously, it intensifies the load transfer between the front and rear wheels, resulting in a safety accident. To improve the carrying capacity and reduce the maintenance cost, leaf spring suspension has been used as the typical suspension system for mining vehicles, but it cannot effectively suppress the vehicle body vibration.

It is known that the vehicle suspension system determines the full vehicle performances, such as ride comfort 
and handling safety [2]. Its properties can be described by using the combination of four suspension modes: bounce, pitch, roll, and warp [3]. For mining vehicles, the dynamic performance primarily depends on the coupled motion between the bounce and pitch modes. Thus, the reduction in vehicle body bounce and pitch motions is crucial to improve the ride comfort and stability of mining vehicles. However, the conventional suspension (CS) cannot well coordinate the bounce and pitch modes of the vehicle body. It is difficult to simultaneously obtain better ride comfort and handling performance by adjusting the suspension modal properties. To solve these problems, the remarkable advantages of active suspension (AS) have attracted the attention of researchers. Many control methods have been proposed to achieve the preferable vehicle performances, such as fuzzy logic control [4], optimal control [5], backstepping control [6], predictive control [7], and adaptive control [8]. Wu et al. [9] designed the active front steering (AFS) system by using the sliding mode control method to effectively improve vehicle handling and stability. Cheng et al. [10] proposed a human-machine cooperative-driving controller with a hierarchical structure to enhance vehicle dynamic stability effectively to ensure the driver's intention. $\mathrm{Li}$ et al. [11] combined the adaptive square-root cubature Kalman filter with the integral correction fusion to acquire the slide-slip angle information for vehicle active safety control. Wong et al. [12] presented a novel integrated controller to coordinate the interactions among the AS, AFS, and direct yaw moment control (DYC), and it could effectively improve the lateral and vertical dynamics of the vehicle. Although these advanced control techniques can preferably solve the contradiction of vehicle performances, some drawbacks regarding control complexity, uncertain reliability, and high cost are inevitable.

To overcome these drawbacks of the CS and AS, a passive interconnected suspension (IS) system can be used alternatively owing to its simplicity, reliability, and zero energy consumption. The IS system between the individual wheel stations is typically realized through mechanical [13], hydraulic [14], and pneumatic [15] methods. The movement of any one of the wheels can generate the forces at other wheel stations. Its remarkable advantage is the passive decoupling of four suspension modes. In other words, this suspension can own the soft stiffness in the bounce and warp modes, and the stiff stiffness in the pitch and roll modes, simultaneously. In recent decades, many research efforts have been focused upon using interconnected suspension systems [16, 17]. Behave [18] developed a parametric study of the influence of pneumatic pitch-plane interconnection arrangements on vehicle ride performance. Yao et al. [19] designed a novel dual-mode interconnected suspension to optimize conflicting vehicle performance requirements by switching different modes. Guo et al. [20] devised a hydropneumatic interconnected suspension to enhance vehicle ride, traction ability, and trafficability. Zhang et al. [21] presented the research on the multibody system dynamics of vehicles fitted with HIS systems, and derived the impedance matrix of hydraulic subsystems for a rollplane HIS system with linear parameters. Cao et al. [17] proposed interconnected hydro-pneumatic suspensions in roll planes and analyzed the dynamic characteristics of the HIS system at the full car level. Ding et al. [22] investigated the roll dynamics of a tri-axle truck model with the HIS system based on the established dynamic equations of motion for the coupled system. Zhu et al. [23] developed an investigation into the road-holding ability of a vehicle equipped with a hydraulically interconnected roll-plane suspension system. Zhou et al. [24] provided the parameter sensitivities of a half-car fitted with the HIS system using a global sensitivity analysis method. Wang et al. [25] proposed a new hydraulically interconnected inertia-spring-damper suspension to coordinate ride comfort and handling stability. The HIS used in the studies above has been proven capable of achieving good dynamic performances compared with the CS.

The vibration property of mining vehicles is easily subject to both the vertical bounce and pitch motions. Therefore, a suppression that can reduce bounce and pitch motions is essential for the suspension design of mining vehicles. A novel HIS system is proposed herein to effectively control the bounce and pitch motions to improve the ride comfort and handling performance. The remainder of the paper is organized as follows. Section 2 presents the description of a HIS-equipped vehicle. The modeling methodology is developed for deriving the dynamic equations of the HIS-equipped vehicle in Section 3. The modal analytical methods of vibration transmissibility and frequency response under stochastic road irregularity inputs are described in Section 4. Section 5 presents the comparative analyses of mining vehicles with the CS and HIS systems. Meanwhile, the sensitivity analysis of loss coefficient for damper valves is also given. Finally, the conclusions are drawn in Section 6.

\section{Description of HIS-equipped Vehicles}

The full mining vehicle studied herein contains two axles that are connected to the vehicle body through leaf spring suspension. A seven-degree-of-freedom (sevenDOF) car model comprising the lumped sprung mass and unsprung mass is used to describe the full mining vehicle. The schematic diagram of a two-axle vehicle with the HIS system is shown in Figure 1. Herein, the sprung mass $m_{s}$ is assumed to be a rigid body with three degrees-of-motion freedom in the vertical, pitch, and roll 


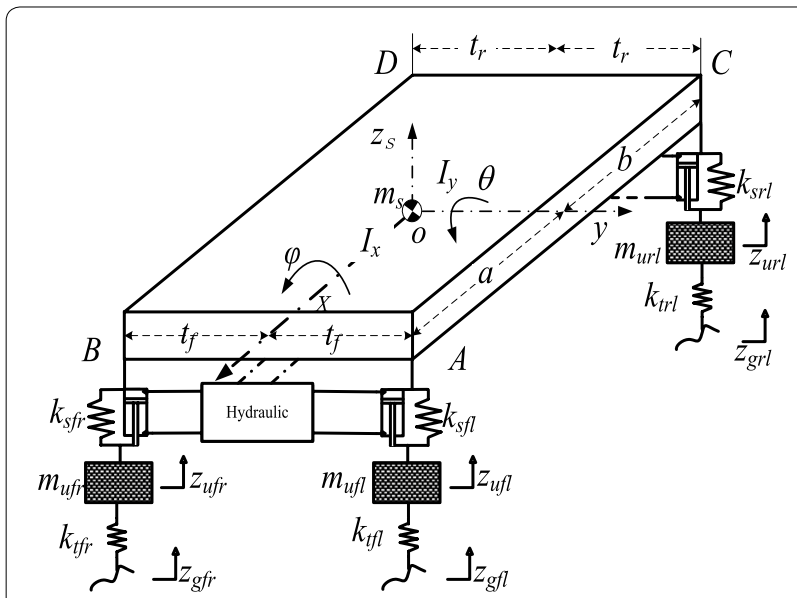

Figure 1 Schematics of a seven-DOF two-axle vehicle model with HIS system

directions $\left(z_{s}, \theta\right.$, and $\left.\phi\right)$. Each unsprung mass $m_{u i}(i=f r$, $f l, r r, r l)$ has a one degree-of-motion freedom in the vertical direction $\left(z_{u i}\right)$. The CG of the vehicle is assumed to be located on a fixed distance $(a$ and $b)$ from the y-axis and a fixed distance $\left(t_{f}\right.$ and $\left.t_{r}\right)$ from the $x$-axis. The stiffness of the suspensions is assumed to be equal to $k_{s i}$, and those of the tires are assumed to be equal to $k_{t i}$, respectively. $z_{g i}$ is the excitation of the road surface exerted on the tire. The pitch inertia $I_{y}$, and the roll inertia $I_{x}$ are obtained about the CG. The subscripts $(f r, f l, r r$, and $r l$ ) are used to denote the front-right, front-left, rear-right, and rear-left, respectively.

Herein, the HIS-equipped vehicle model is a mechanical and hydraulic coupled system. The layout for the hydraulic subsystem used in the HIS-equipped vehicle model is shown in Figure 2. The shock absorbers of the CS are replaced by the double acting actuators of the HIS that can directly provide the forces on the vehicle body and wheel assemblies. In the hydraulic subsystem, the hydraulic actuator has a cylinder and a piston, and the cylinder can be divided into a top and a bottom chamber. Furthermore, all the accumulators are integrated into the top chambers. The top chambers include some pin holes, namely accumulator valves, to permit the flow communication between the accumulators and top chambers. These valves can cause the differential pressure in the corresponding chambers. This structure is defined as a pneumatic-hydraulic actuator, which can rapidly alleviate the vibration from road excitations. The chambers of actuator assemblies are interconnected by the hydraulic circuits to enable the flow movement among the chambers, accumulators, and damper valves.

The wheel assemblies are coupled to each other by the HIS system. In the body bounce motion shown in

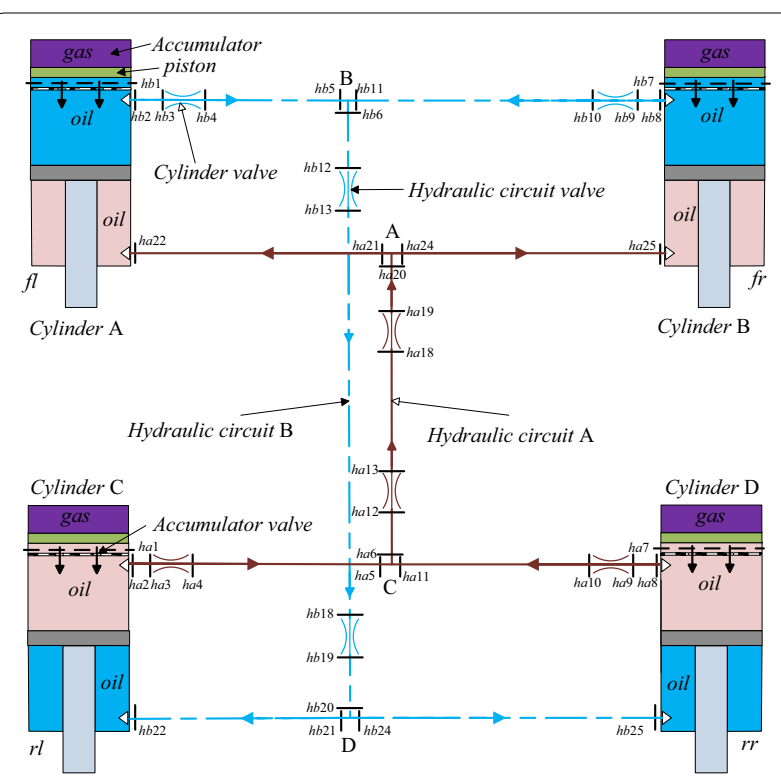

Figure 2 Layout for the hydraulic subsystem

Figure 1, the top and bottom chambers are compressed and extended, respectively. Owing to the asymmetry of the hydraulic cylinders, every hydraulic circuit contains a small amount of fluid flowing into the accumulators. This leads to a small pressure change in the two hydraulic circuits. Thus, the actuators could generate an additional hydraulic force to restrict the bounce motion of the sprung mass relative to the unsprung mass. In the body pitch motion, all the front and rear suspensions are compressed and extended, respectively. The front and rear cylinder bodies move downward and upward relative to the piston rod, respectively. It reduces the volume of the front top and rear bottom chambers, and simultaneously increases the volume of the front bottom and rear top chambers. Thus, the fluid in circuit B flows into the corresponding accumulator $\mathrm{B}$, and the fluid in the corresponding accumulator A flows into circuit $\mathrm{A}$. Consequently, the pressures in circuits $\mathrm{A}$ and $\mathrm{B}$ are increased and decreased, respectively. It in return generates the large pitch restoring moment to prevent the vehicle body pitch motion relative to the unsprung mass.

\section{Model Development of Vehicles with HIS}

Based on the free body diagram method [22] and by regarding the hydraulic forces as external forces, the equations of motion for the integrated mechanicalhydraulic system can be derived as

$$
\boldsymbol{M} \ddot{\boldsymbol{y}}+\boldsymbol{C} \dot{\boldsymbol{y}}+\boldsymbol{K} \boldsymbol{y}=\boldsymbol{F}_{H}+\boldsymbol{f}_{\mathrm{e}},
$$

where $M, C$, and $K$ are the mass, damping, and stiffness matrices for the mechanical system, respectively. $\boldsymbol{y}=$ 
$\left[z_{s} \theta \phi z_{u f l} z_{u f r} z_{u r l} z_{u r r}\right]^{\mathrm{T}}$ is the displacement vector, $\boldsymbol{f}_{e}$ is the external force vector. The hydraulic force matrix $\boldsymbol{F}_{H}$ is defined as $\boldsymbol{F}_{\boldsymbol{H}}=\boldsymbol{D}_{1} \boldsymbol{A}_{c} \boldsymbol{P}$. The pressure vector $\boldsymbol{P}$ related to the pressures $p_{i T}$ and $p_{i B}(i=f r, f l, r r, r l)$ in the corresponding cylinder chambers is given as $\boldsymbol{P}=\left[p_{f l T} p_{f l B}\right.$ $\left.p_{f r T} p_{f r B} p_{r l T} p_{r l B} p_{r r T} p_{r r B}\right]^{\mathrm{T}}$, the subscript symbols $T$ and $B$ denote the top and bottom chambers, respectively. $A_{c}$ $=\left[A_{f l T} A_{f l B} A_{f r T} A_{f r B} A_{r l T} A_{r l B} A_{r r T} A_{r r B}\right]^{\mathrm{T}}$ is the area matrix corresponding to the top chamber area $A_{i \mathrm{~T}}$ and the bottom chamber area $A_{i B}$, respectively. $D_{1}$ is the linear coefficient matrix.

For a double-acting hydraulic actuator, the relative velocity between the cylinder body and the piston rod results in a boundary fluid flow towards the mechanical-fluid interface. The relationship between the relative velocity and fluid flow forms the primary component of the boundary flow. Owing to small-amplitude oscillations under low pressure, the compression of the fluid and the cross-line leakage in the cylinder chambers can be ignored. Figure 3 shows the boundary flow conditions for the hydraulic actuator model.

In the HIS system, the boundary flow conditions for the double-acting actuators are written as follows:

$$
\begin{gathered}
q_{f l T}=q_{f l s T}+q_{f l d}, \quad q_{f l B}=q_{f l s B}, \\
q_{f r T}=q_{f r s T}+q_{f r d}, \quad q_{f r B}=q_{f r s B}, \\
q_{r l T}=q_{r l s T}+q_{r l d}, \quad q_{r l B}=q_{r l s B}, \\
q_{r r T}=q_{r r s T}+q_{r r d}, \quad q_{r r B}=q_{r r s B},
\end{gathered}
$$

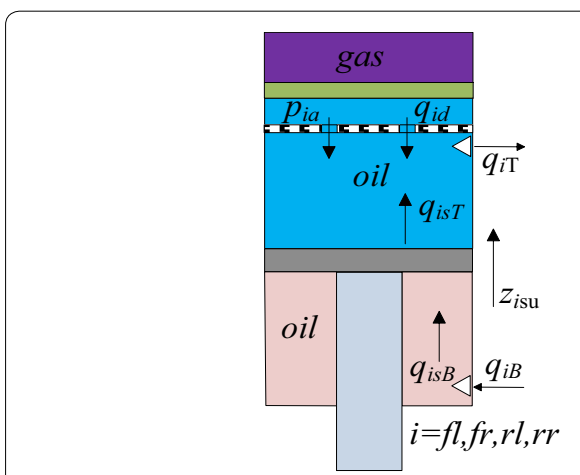

Figure 3 Double-acting cylinder model where $q_{i \mathrm{~T}}$ and $q_{i \mathrm{~B}}$ are the out-flow and in-flow in the corresponding chambers, respectively. $q_{i s T}=A_{i T} \dot{z}_{i s u}$ and $q_{i s B}$ $=A_{i B} \dot{z}_{i s u}$ are volumetric flows generated by the relative velocity $\dot{z}_{i s u}$ and piston area $A_{i T} / A_{i B}$ in the corresponding chambers. The pinhole flow $q_{i d}$ is assumed to be proportional to the pressure difference between the accumulators and top chambers, given by $q_{i d}=\left(p_{i \mathrm{a}}-p_{i \mathrm{~T}}\right) / R_{i a v}$. The pressure in the corresponding accumulators is defined as $p_{i a}$. The loss coefficient $R_{i a v}$ of the accumulator valve is written as $R_{i a v}=128 \mu l_{0} / \pi d_{a v}^{2}$ in Ref. [26], where $l_{0}$ and $d_{a v}$ represent the length and diameter of the accumulator valve, respectively.

As shown, the velocity vector $\dot{z}_{s-u}=\left[\dot{z}_{f l s u} \dot{z}_{f r s u} \dot{z}_{r l s u} \dot{z}_{r r s u}\right]^{\mathrm{T}}$ is relative to the velocity vector $\dot{\boldsymbol{y}}$ of the lumped sprung mass and unsprung mass, given by $\dot{\boldsymbol{z}}_{s-u}=\boldsymbol{D}_{2} \dot{\boldsymbol{y}}$, where $\boldsymbol{D}_{2}$ is the linear coefficient matrix. Applying these relationships to the four actuator cylinders yields

$$
\boldsymbol{Q}=\boldsymbol{A}_{c} \boldsymbol{D}_{2} \dot{\boldsymbol{y}}+\boldsymbol{D}_{v 1} \boldsymbol{P}+\boldsymbol{D}_{v 2} \boldsymbol{P}_{a},
$$

where the flow vector is $\boldsymbol{Q}=\left[q_{f l T} q_{f l B} q_{f r T} q_{f r B} q_{r l T} q_{r l B} q_{r r T}\right.$ $\left.q_{r r B}\right]^{\mathrm{T}}$, and the accumulator pressure vector $\boldsymbol{P}_{a}=\left[p_{f l a}\right.$ $\left.p_{\text {fra }} p_{r l a} p_{r r a}\right]^{\mathrm{T}} . \boldsymbol{D}_{v 1}$ and $\boldsymbol{D}_{v 2}$ are the resistance coefficient matrices of the cylinder pressure and accumulator pressure, respectively.

To obtain the motion properties of the mechanical-fluid coupled system in Eq. (1), the relationship between flow $\boldsymbol{Q}$ and pressure $\boldsymbol{P}$ of the fluid system must be derived. In this study, the transfer impedance matrix method is employed to describe the relationship between the flow rate $\boldsymbol{Q}$ and pressure $\boldsymbol{P}$, given as $\boldsymbol{Q}=\boldsymbol{T} \boldsymbol{P}$, where $T$ is the impedance matrix of the hydraulic subsystem determined by the impedance matrices of the fluid components. As shown in Figure 2, the fluid components primarily include five types of components, namely hydraulic actuators, piston accumulators, three-way junctions, damper valves, and fluid pipelines. For the HIS system, the connection sections of different fluid components are marked by hai $(i=1,2, \ldots, 25)$ and $h b i$, respectively. The state vector composed of the pressure and flow rate $(p$ and $q)$ at the section $h j i(j=a, b)$ is defined as $\boldsymbol{x}_{i j}=\left[\begin{array}{ll}p & q\end{array}\right]_{h i j}^{\mathrm{T}}$ The transmission matrix $\boldsymbol{N}^{k}$ is employed to describe the relationship between state vectors $\boldsymbol{x}_{i j}$ and $x_{i j+1}$ of the two terminal sections for the $k$ th component, given as $\boldsymbol{x}_{j i+1}=\boldsymbol{N}^{k} \boldsymbol{x}_{j i}(k=P, V)$, where the details of the transfer matrix $N^{k}$ for these fluid components can be found in Ref. [21].

The connectivity matrix $N$ can be obtained by a sequence of multiplications of the involved component impedance matrices according to the theory of transfer matrix. In the hydraulic circuit B of the HIS system, the connectivity matrix $N$ can be written as 


$$
\begin{aligned}
& \underbrace{\left[\begin{array}{ll}
N_{11} & N_{12} \\
N_{21} & N_{22}
\end{array}\right]}_{N_{h b 2} \rightarrow h b 5}=\boldsymbol{N}_{h b 4 \rightarrow h b 5}^{P} \boldsymbol{N}_{h b 3 \rightarrow h b 4}^{V} \boldsymbol{N}_{h b 2 \rightarrow h b 3}^{P}, \\
& \underbrace{\left[\begin{array}{ll}
N_{11} & N_{12} \\
N_{21} & N_{22}
\end{array}\right]}_{N_{h b 8 \rightarrow h b 11}}=\boldsymbol{N}_{h b 10 \rightarrow h b 11}^{P} \boldsymbol{N}_{h b 9 \rightarrow h b 10}^{V} \boldsymbol{N}_{h b 8 \rightarrow h b 9}^{P}, \\
& \underbrace{\left[\begin{array}{ll}
N_{11} & N_{12} \\
N_{21} & N_{22}
\end{array}\right]}_{N_{h b 21 \rightarrow h b 22}}=\boldsymbol{N}_{h b 21 \rightarrow h b 22}^{P}, \underbrace{\left[\begin{array}{ll}
N_{11} & N_{12} \\
N_{21} & N_{22}
\end{array}\right]}_{N_{h b 24 \rightarrow h b 25}}=\boldsymbol{N}_{h b 24 \rightarrow h b 25}^{P},
\end{aligned}
$$

The relationships of pressures and flows for the threeway junctions $B$ and $D$, through applying the continuity equation and assuming no pressure loss, can be defined as follows:

$$
\left\{\begin{array}{l}
q_{h b 5}+q_{h b 11}=q_{h b 6}, p_{h b 5}=p_{h b 6}=p_{h b 11}, \\
q_{h b 21}+q_{h b 24}=q_{s b 20}, p_{h b 20}=p_{h b 21}=p_{h b 24} .
\end{array}\right.
$$

Combining Eqs. (4)-(9), the impedance matrix $\boldsymbol{T}_{b}$ is obtained by solving the symbolic equations and given as

$$
\underbrace{\left[\begin{array}{ll}
N_{11} & N_{12} \\
N_{21} & N_{22}
\end{array}\right]}_{N_{h b 6 \rightarrow h b 20}}=\boldsymbol{N}_{h b 19 \rightarrow h b 20}^{P} \boldsymbol{N}_{h b 18 \rightarrow h b 19}^{V} \boldsymbol{N}_{h b 13 \rightarrow h b 18}^{P} \boldsymbol{N}_{h b 12 \rightarrow h b 13}^{V} \boldsymbol{N}_{h b 6 \rightarrow h b 12}^{P},
$$

where the rear subscripts in the matrices above represent the inflow and outflow sections of the corresponding components, respectively. The front subscripts $P$ and $V$ refer to the components of fluid pipes and valves, respectively. Accordingly, the relationships between the state vectors at all sections mentioned in Eqs. (4)-(7) are described as

$$
\underbrace{\left[\begin{array}{c}
p_{h b 5} \\
q_{h b 5}
\end{array}\right]}_{x_{h b 5}}=N_{h b 2 \rightarrow h b 5} \underbrace{\left[\begin{array}{c}
p_{h b 2} \\
q_{h b 2}
\end{array}\right]}_{x_{h b 2}}
$$

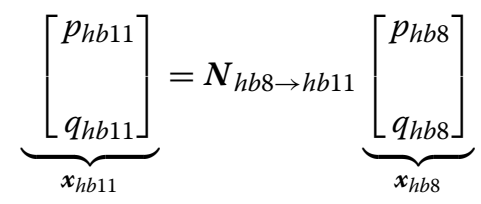

$$
\underbrace{\left[\begin{array}{c}
p_{h b 22} \\
q_{h b 22}
\end{array}\right]}_{x_{h b 22}}=N_{h b 21 \rightarrow h b 22} \underbrace{\left[\begin{array}{c}
p_{h b 21} \\
q_{h b 21}
\end{array}\right]}_{x_{h b 21}} \underbrace{\left[\begin{array}{c}
p_{h b 25} \\
q_{h b 25}
\end{array}\right]}_{x_{h b 25}}=N_{h b 24 \rightarrow h b 25} \underbrace{\left[\begin{array}{c}
p_{h b 24} \\
q_{h b 24}
\end{array}\right]}_{x_{h b 24}}
$$

$$
\underbrace{\left[\begin{array}{c}
p_{h b 20} \\
q_{h b 20}
\end{array}\right]}_{x_{h b 20}}=N_{h b 6 \rightarrow h b 20} \underbrace{\left[\begin{array}{c}
p_{h b 6} \\
q_{h b 6}
\end{array}\right]}_{x_{h b 6}}
$$




$$
\left[\begin{array}{c}
q_{h b 2} \\
q_{h b 8} \\
q_{h b 22} \\
q_{h b 25}
\end{array}\right]=\underbrace{\left[\begin{array}{llll}
T_{b 11} & T_{b 12} & T_{b 13} & T_{b 14} \\
T_{b 21} & T_{b 22} & T_{b 23} & T_{b 24} \\
T_{b 31} & T_{b 32} & T_{b 33} & T_{b 34} \\
T_{b 41} & T_{b 42} & T_{b 43} & T_{b 44}
\end{array}\right]}_{T_{b}}\left[\begin{array}{c}
p_{h b 2} \\
p_{h b 8} \\
p_{h b 22} \\
p_{h b 25}
\end{array}\right] .
$$

Similarly, the impedance matrix $\boldsymbol{T}_{A}$ of the hydraulic circuit $A$ can also be obtained. Thus, the impedance matrices $\boldsymbol{T}_{A}$ and $\boldsymbol{T}_{b}$ can be combined and expressed as

$$
Q=\underbrace{\left[\begin{array}{cccccccc}
T_{b 11} & 0 & T_{b 12} & 0 & 0 & T_{b 13} & 0 & T_{b 14} \\
0 & T_{a 31} & 0 & T_{a 32} & T_{a 33} & 0 & T_{a 34} & 0 \\
T_{b 21} & 0 & T_{b 22} & 0 & 0 & T_{b 23} & 0 & T_{b 24} \\
0 & T_{a 41} & 0 & T_{a 42} & T_{a 43} & 0 & T_{a 34} & 0 \\
0 & T_{a 11} & 0 & T_{a 12} & T_{a 13} & 0 & T_{a 14} & 0 \\
T_{b 31} & 0 & T_{b 32} & 0 & 0 & T_{b 33} & 0 & T_{b 34} \\
0 & T_{a 21} & 0 & T_{a 22} & T_{a 23} & 0 & T_{a 24} & 0 \\
T_{b 41} & 0 & T_{b 42} & 0 & 0 & T_{b 43} & 0 & T_{b 44}
\end{array}\right]}_{T} \boldsymbol{P},
$$

where $\boldsymbol{T}$ is the impedance matrix of the HIS system. As shown, the impedance matrix $\boldsymbol{T}$ is determined by the connectivity matrices and related to the precise arrangement of the component elements in hydraulic circuits.
Furthermore, $N_{a c}$ is defined as the impedance of the accumulator. Thus, the accumulator pressure vector can be written as

$$
\boldsymbol{P}_{a}=\left[\begin{array}{llll}
N_{a c} & N_{a c} & N_{a c} & N_{a c}
\end{array}\right]^{\mathrm{T}} \boldsymbol{q}_{d}^{\mathrm{T}},
$$

where $\boldsymbol{q}_{d}$ is the accumulator fluid vector, given as $\boldsymbol{q}_{d}=$ $\left[q_{\text {fld }} q_{\text {frd }} q_{r l d} q_{r r d}\right]^{\mathrm{T}}$. Owing to the different pressures inside the accumulators and top chambers generated by the accumulator valves, the fluid-flows $q_{f l d}, q_{f r d}, q_{r l d}$, and $q_{\text {rrd }}$ at sections $h b 1, h a 1, h b 7$, and $h a 7$ are derived as

$$
\begin{gathered}
q_{f l d}=\left(N_{a c} q_{f l d}-p_{h b 2}\right) / R_{f l a v}, q_{f r d}=\left(N_{a c} q_{f r d}-p_{h b 8}\right) / R_{f r a v}, \\
q_{r l d}=\left(N_{a c} q_{r l d}-p_{h a 2}\right) / R_{r l a v}, \quad q_{r r d}=\left(N_{a c} q_{r r d}-p_{h a 8}\right) / R_{r r a v} .
\end{gathered}
$$

According to Eq. (13), the relationships between accumulator fluid vector $\boldsymbol{q}_{d}$ and pressure vector $\boldsymbol{P}$ can be rewritten as $\boldsymbol{q}_{d}=\boldsymbol{D}_{v} \boldsymbol{P}$. $\boldsymbol{D}_{v}$ is the resistance coefficient matrix related to the impedance $N_{a c}$ and the loss coefficient $R_{\text {iav }}$. Based on the Laplace transform of Eq. (11) with zero initial conditions, substituting it into Eq. (3) yields

$$
\boldsymbol{P}=s\left(\boldsymbol{T}-\boldsymbol{D}_{v}\right)^{-1} \boldsymbol{A}_{c} \boldsymbol{D}_{2} \boldsymbol{Y}(s) .
$$

Combining Eqs. (1) and (14), the equation for the coupled system is expressed in the state-space form as

$$
s \boldsymbol{Z}(s)=\boldsymbol{A}(s) \boldsymbol{Z}(s)+\boldsymbol{B} \boldsymbol{F}(s),
$$

where the system state vector is $\boldsymbol{z}=\left[\begin{array}{l}\boldsymbol{y} \\ \boldsymbol{y}\end{array}\right]^{\mathrm{T}}$ and $\boldsymbol{Z}(s)$ is the Laplace transformation of $\boldsymbol{z}, \boldsymbol{F}(s)$ is the Laplace transformation of the external force vector $\boldsymbol{f}_{\boldsymbol{e}}, \boldsymbol{A}(s)$ is the system characteristic matrix, and $\boldsymbol{B}$ is the input coefficient matrix. They are described in the following form:

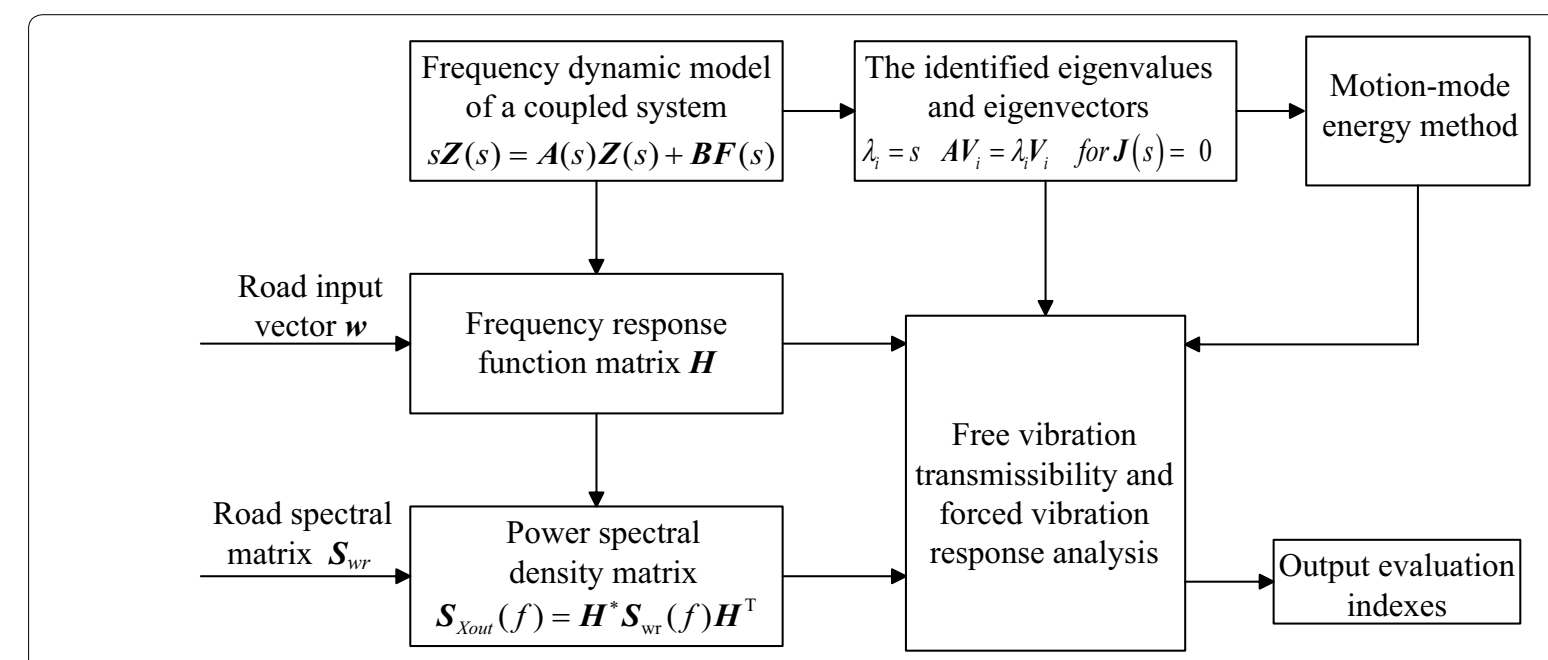

Figure 4 Analysis process of the vehicle vibration responses 


$$
A(s)=\left[\begin{array}{rr}
\boldsymbol{O} & \boldsymbol{I} \\
-\boldsymbol{M}^{-1} \boldsymbol{K} & -\boldsymbol{M}^{-1} \hat{\boldsymbol{C}}(s)
\end{array}\right], \quad \boldsymbol{B}=\left[\begin{array}{r}
\boldsymbol{O} \\
\boldsymbol{M}^{-1}
\end{array}\right] .
$$

In Eq. (16), $\hat{C}=C+C_{h}$ is the complex damping matrix. $\boldsymbol{C}_{h}=\boldsymbol{D}_{1} \boldsymbol{A}_{c}\left(\boldsymbol{T}-\boldsymbol{D}_{v}\right)^{-1} \boldsymbol{A}_{c} \boldsymbol{D}_{2}$ is the damping contribution from hydraulic subsystem.

\section{Vehicle Vibration Characteristics Analysis}

The modal analysis process is summarized briefly in the block diagram, as shown in Figure 4. Eq. (15) represents the general equation of motions for the completely coupled system in the frequency domain. The frequency response function (FRF) and power spectral density (PSD) response can be derived by the frequency dynamic model of the coupled system to evaluate the vehicle performances. Meanwhile, the eigenvalues and eigenvectors are identified to obtain the natural frequency and mode shape of the coupled system. The motion-mode energy method [27] derived by the identified eigenvalues and eigenvectors is employed to analyze the vehicle dynamic characteristics in the frequency domain.

As shown, the system modal characteristic matrix $\boldsymbol{A}(s)$ in Eq. (15) is frequency dependent and this matrix changes with the input frequency $s$. Based on the definition of the eigenvalue, solutions of the equation $\mid \operatorname{det}(\boldsymbol{A}(s)$ $-s \boldsymbol{I}) \mid=0$ are the eigenvalues of $\boldsymbol{A}(s)$. Owing to the large numerical values of some of the parameters, the exact eigenvalues of $\boldsymbol{A}(s)$ are difficult to obtain by numerical means. Thus, the approximate roots are acceptable if the function $\boldsymbol{J}(s)=|\operatorname{det}(\boldsymbol{A}(s)-s \boldsymbol{I})|$ is approaching a local minimum value in the vicinity of $s$. To obtain the modal parameters, the Matlab optimization library function fminsearh was used to identify the real eigenvalues and eigenvectors according to the local minimum values.

Using the optimization method, the complex eigenvalues $\lambda_{i}$ and eigenvectors $V_{i}$ resulting from the solutions of the equation $|\operatorname{det}(\boldsymbol{A}(s)-\boldsymbol{s} \boldsymbol{I})|=0$ are given as

$$
\lambda_{i}=s, \boldsymbol{A} \boldsymbol{V}_{i}=\lambda_{i} \boldsymbol{V}_{i}, \text { for } \boldsymbol{J}(s)=0,
$$

where the first seven terms of $\boldsymbol{V}_{i}(i=1,2, \ldots, 7)$ represent the mode shape of the displacement vector. For each complex root $\lambda_{i}=a_{i}+j b_{i}$, the natural frequency $f_{i}$ and damping ratio $\xi_{i}$ for each mode are given as $f_{i}=\operatorname{sqrt}\left(a^{2}{ }_{i}+b^{2}{ }_{i}\right) / 2 \pi$ and $\xi_{i}=a_{i} / \operatorname{sqrt}\left(a_{i}^{2}+b_{i}^{2}\right)$.

The modal transition matrix can be determined by the eigenvalue matrix and eigenvector matrix, and it can be defined as

$$
\boldsymbol{L}=\left[\begin{array}{ll}
\boldsymbol{\Psi} & \boldsymbol{\Lambda} \boldsymbol{\Psi}
\end{array}\right]_{14 \times 7}^{\mathrm{T}}
$$

where $\psi=\operatorname{diag}\left[\begin{array}{lllllll}\lambda_{1} & \lambda_{2} & \lambda_{3} & \lambda_{4} & \lambda_{5} & \lambda_{6} & \lambda_{7}\end{array}\right]$ is the eigenvalue matrix and $\boldsymbol{\Lambda}=\left[\begin{array}{lllllll}\boldsymbol{V}_{1} & \boldsymbol{V}_{2} & \boldsymbol{V}_{3} & \boldsymbol{V}_{4} & \boldsymbol{V}_{5} & \boldsymbol{V}_{6} & \boldsymbol{V}_{7}\end{array}\right]$ is the eigenvector matrix.

The system state vector $\boldsymbol{Z}$ can be described as a superposition of the motion-modes through modal transformation as

$$
\boldsymbol{Z}=\boldsymbol{L} \boldsymbol{u}(f)
$$

where $\boldsymbol{u}=\left[\begin{array}{lllllll}u_{1} & u_{2} & u_{3} & u_{4} & u_{5} & u_{6} & u_{7}\end{array}\right]^{\mathrm{T}}$ is a modal amplitude vector. As shown, the modal amplitude $u_{i}$ is a complex number that contains both a scalar quantity and the phase information of this mode in the frequency domain.

As $\boldsymbol{L}$ is a known matrix determined by the coupled mechanical-hydraulic system, the modal amplitude vector $\boldsymbol{u}(f)$ can be obtained by applying the least-squares method, and the form of $\boldsymbol{u}$ is written as

$$
\boldsymbol{u}(f)=\left(\boldsymbol{L}^{\mathrm{T}} \boldsymbol{L}\right)^{-1} \boldsymbol{L}^{\mathrm{T}} \boldsymbol{Z} .
$$

Subsequently, the modal amplitude vector $\boldsymbol{u}$ must be transferred into a physical coordinate as $\left[\boldsymbol{d}_{i} \dot{\boldsymbol{d}}_{i}\right]$, where $\boldsymbol{d}_{i}=\operatorname{real}\left(u_{i} V_{i}\right), \dot{\boldsymbol{d}}_{i}=\operatorname{real}\left(u_{i} V_{i} \lambda_{i}\right)$, and $\boldsymbol{d}_{i}$ can be written as $\boldsymbol{d}_{i}=\left[\boldsymbol{d}_{m i} \boldsymbol{d}_{u i}\right]$ referring to the displacement vector $\boldsymbol{y}$ defined in Eq. (1). In this study, $\boldsymbol{d}_{m i}$ and $\boldsymbol{d}_{u i}$ are used to describe the body and wheel motions, respectively. The vector $\left[\boldsymbol{d}_{i} \dot{\boldsymbol{d}}_{i}\right]$ is the corresponding state vector component in the form of the displacement and velocity, and represents the $i$ th motion-mode's contribution to the vehicle's body and wheel motions. The energy in one motion-mode does not exchange with the others according to the orthogonal property of modal transformation, and its energy is continuously converted between the potential energy and kinetic energy, and decays owing to the damping effect. The kinetic energy $\boldsymbol{e}_{k i}$, potential energy $\boldsymbol{e}_{p i}$, and the sum of mechanical energy $\boldsymbol{e}_{i}$ for each motion-mode stored in the $i$ th mode can be given as

$$
\begin{array}{r}
\boldsymbol{e}_{k i}=\frac{1}{2} \dot{\boldsymbol{d}}_{i}^{\mathrm{T}} \boldsymbol{M} \dot{\boldsymbol{d}}_{i}, \quad \boldsymbol{e}_{p i}=\frac{1}{2}\left[\begin{array}{r}
\boldsymbol{D}_{3} \boldsymbol{d}_{m i}-\boldsymbol{d}_{u i} \\
\boldsymbol{d}_{u i}-\boldsymbol{d}_{g i}
\end{array}\right]^{\mathrm{T}} \boldsymbol{D}_{k}\left[\begin{array}{r}
\boldsymbol{D}_{3} \boldsymbol{d}_{m i}-\boldsymbol{d}_{u i} \\
\boldsymbol{d}_{u i}-\boldsymbol{d}_{g i}
\end{array}\right], \\
\boldsymbol{e}_{i}=\boldsymbol{e}_{k i}+\boldsymbol{e}_{p i},
\end{array}
$$


where $D_{k}=\operatorname{diag}\left[k_{s f l} k_{s f r} k_{s r l} k_{s r r}\right]^{\mathrm{T}}$ and $\boldsymbol{d}_{g i}$ is the projection of the road input in the same coordinate frame with the vehicle motion-mode, which can be determined by the same principle of modal transformation. $\boldsymbol{D}_{3}$ is the coefficient matrix related to the physical parameters of the vehicle model.

In this study, the FRF is employed to analyze the vibration characteristics of the suspension system in the frequency domain. The suspension vibration performances can be evaluated using the displacement response amplitude transmissibility between the displacement vector $y$ and road input vector $\boldsymbol{w}=\left[\begin{array}{llllll}0 & 0 & 0 & z_{g f l} & z_{g f r} & z_{g r l} \\ z_{g r l}\end{array}\right]^{\mathrm{T}}$. Combining Eqs. (1) and (20), the FRF matrix $\boldsymbol{H}_{\text {dis }}$ from the road input vector $\boldsymbol{w}$ to the displacement output vector $\boldsymbol{y}$ is described as

$$
\boldsymbol{H}_{\text {dis }}(s)=\frac{\boldsymbol{Y}(s)}{\boldsymbol{W}(s)}=\left(s^{2} \boldsymbol{M}+s \hat{\boldsymbol{C}}(s)+\boldsymbol{K}\right)^{-1} \boldsymbol{\beta}_{7 \times 7}(s),
$$

where $s=j 2 \pi f$ and $W(s)$ is the Laplace transformation of the road input vector $\boldsymbol{w}$, and $\boldsymbol{\beta}(s)$ is a $7 \times 7$ input coefficient matrix, given as $\beta(s)=\operatorname{diag}\left[\begin{array}{lllll}0 & 0 & 0 & k_{t f l} & k_{t f r} \\ k_{t r l} & k_{t r r}\end{array}\right]$. The FRF matrix is used to describe the system displacement response to any harmonic road excitation in the frequency domain.

To evaluate ride comfort, the corresponding transfer functions of the vibration accelerations, suspension deflections, and tire dynamic forces, which are related to the road input vector $W(s)$, are defined as follows, respectively:

$$
\begin{array}{r}
\boldsymbol{H}_{a c c}=\frac{s^{2} \boldsymbol{Y}(s)}{\boldsymbol{W}(s)}=s^{2} \boldsymbol{H}_{d i s}, \\
\boldsymbol{H}_{\text {sus }}=\frac{\boldsymbol{D}_{2} \boldsymbol{Y}(s)}{\boldsymbol{W}(s)}=\boldsymbol{D}_{2} \boldsymbol{H}_{\text {dis }}, \\
\boldsymbol{H}_{\text {tire }}=\frac{\boldsymbol{\beta}(s)(\boldsymbol{Y}(s)-\boldsymbol{W}(s))}{\boldsymbol{W}(s)}=\boldsymbol{\beta}(s)\left(\boldsymbol{H}_{\text {dis }}-\boldsymbol{I}\right),
\end{array}
$$

where the Laplace transformations of the vibration accelerations, suspension deflections, and tire dynamic forces can be defined as $\boldsymbol{X}_{a c c}=s^{2} \boldsymbol{Y}(s), \boldsymbol{X}_{s u s}=\boldsymbol{D}_{2} \boldsymbol{Y}(s)$, and $\boldsymbol{X}_{\text {tire }}=\boldsymbol{\beta}(s)(\boldsymbol{Y}(s)-\boldsymbol{W}(s))$, respectively.

To obtain the evaluation outputs $X_{\text {out }}=\left[\begin{array}{ll}X_{a c c} & X_{\text {sus }}\end{array}\right.$ $\boldsymbol{X}_{\text {tire }}{ }^{\mathrm{T}}$, the entities of all transfer functions are assembled as follows:

$$
\underbrace{\left[\begin{array}{c}
\boldsymbol{X}_{\text {acc }} \\
\boldsymbol{X}_{\text {sus }} \\
\boldsymbol{X}_{\text {tire }}
\end{array}\right]}_{\boldsymbol{X}_{\text {out }}}=\left[\begin{array}{c}
\boldsymbol{H}_{\text {acc }} \\
\boldsymbol{H}_{\text {sus }} \\
\boldsymbol{H}_{\text {tire }}
\end{array}\right] \boldsymbol{W}(s)=\underbrace{\left[\begin{array}{c}
\boldsymbol{H}_{\text {acc }} \\
\boldsymbol{H}_{\text {sus }} \\
\boldsymbol{H}_{\text {tire }}
\end{array}\right] \boldsymbol{D}_{T} \boldsymbol{W}_{r}(s),}_{\boldsymbol{H}}
$$

where $W_{r}(s)$ is the Laplace transformation of the reduced road displacement vector $\boldsymbol{w}_{r}=\left[z_{g f l} z_{g f r} z_{g r l} z_{g r l}\right]^{\mathrm{T}}, \boldsymbol{D}_{T}$ is the transfer coefficient matrix that can describe the corresponding relationship between the reduced road displacement vector $\boldsymbol{w}_{r}$ and the road input vector $\boldsymbol{w}$, given as $\boldsymbol{w}=\boldsymbol{D}_{T} \mathbf{w}_{r}$, and $\boldsymbol{H}$ is defined as the transfer function from inputs $W_{r}(s)$ to the evaluation outputs.

The PSD matrix $\boldsymbol{S}_{\text {Xout }}(f)$ can be derived from the complex transfer function, and the detailed expression is defined as

$$
\boldsymbol{S}_{\text {Xout }}(f)=\boldsymbol{H}^{*} \boldsymbol{S}_{w r}(f) \boldsymbol{H}^{\mathrm{T}},
$$

where $\boldsymbol{H}^{*}$ and $\boldsymbol{H}^{\mathrm{T}}$ are the complex conjugate matrix and transpose matrix, respectively. $S_{w r}(f)$ is the spectral density matrix at four tire-road contact points, given by

$$
S_{w r}(f)=\left[\begin{array}{llll}
S_{d} & S_{r} & S_{d} e^{-j 2 \pi f l / v} & S_{r} e^{-j 2 \pi f l / v} \\
S_{r} & S_{d} & S_{r} e^{-j 2 \pi f l / v} & S_{d} e^{-j 2 \pi f l / v} \\
S_{d} e^{j 2 \pi f l / v} & S_{r} e^{j 2 \pi f l / v} & S_{d} & S_{r} \\
S_{r} e^{j 2 \pi f l / v} & S_{d} e^{j 2 \pi f l / v} & S_{r} & S_{d}
\end{array}\right]
$$

where $l$ is the wheel base, and $v$ is the vehicle velocity. $S_{d}$ and $S_{r}$ are the auto-spectral density and cross-spectral

Table 1 Physical parameters of a seven-DOF vehicle model

\begin{tabular}{ll}
\hline Parameter & Value \\
\hline Vehicle sprung mass $m_{s}(\mathrm{~kg})$ & 3860 \\
Pitch moment inertia of sprung mass $I_{x}\left(\mathrm{~kg} \cdot \mathrm{m}^{2}\right)$ & 8400 \\
Roll moment inertia of sprung mass $I_{y}\left(\mathrm{~kg} \cdot \mathrm{m}^{2}\right)$ & 1400 \\
Distance from sprung mass CG to front axle $a(\mathrm{~m})$ & 1.64 \\
Distance from sprung mass CG to rear axle $b(\mathrm{~m})$ & 1.49 \\
Half width of front axle $t_{f}(\mathrm{~m})$ & 0.32 \\
Half width of rear axle $t_{r}(\mathrm{~m})$ & 0.46 \\
Front unsprung mass $m_{u f}(\mathrm{~kg})$ & 200 \\
Rear unsprung mass $m_{u r}(\mathrm{~kg})$ & 260 \\
Spring rate of front suspension $k_{p s f}(\mathrm{~N} / \mathrm{mm})$ & 265 \\
Spring rate of rear suspension $k_{p s r}(\mathrm{~N} / \mathrm{mm})$ & 265 \\
Damping coefficient of front suspension $c_{p s f}(\mathrm{~N} \cdot \mathrm{m} / \mathrm{s})$ & 4880 \\
Damping coefficient of rear suspension $c_{p s r}(\mathrm{~N} \cdot \mathrm{m} / \mathrm{s})$ & 4880 \\
Spring rate of front suspension $k_{b s f}(\mathrm{~N} / \mathrm{mm})$ & 129.2 \\
Spring rate of rear suspension $K_{\text {bsf }}(\mathrm{N} / \mathrm{mm})$ & 167.4 \\
Vertical stiffness of tire $k_{t f} / k_{t r}(\mathrm{~N} / \mathrm{mm})$ & 1172 \\
\hline
\end{tabular}


Table 2 Hydraulic system parameters

\begin{tabular}{ll}
\hline Parameter & Value \\
\hline Hydraulic fluid density $\left(\mathrm{kg} / \mathrm{m}^{3}\right)$ & 870 \\
Bulk modulus $(\mathrm{MPa})$ & 1400 \\
Accumulator pre-charge gas volume (L) & 0.25 \\
Accumulator pre-charge pressure (bar) & 10 \\
Diameter of cylinder piston (m) & 0.05 \\
Diameter of cylinder piston rod (m) & 0.036 \\
Mean system pressure $P_{0}($ bar) & 23 \\
Loss coefficient of cylinder valve $R_{C V}\left(\mathrm{GPa} \cdot \mathrm{s} / \mathrm{m}^{3}\right)$ & 1.5 \\
Loss coefficient of hydraulic circuit valve $R_{H C V}\left(\mathrm{GPa} \cdot \mathrm{s} / \mathrm{m}^{3}\right)$ & 0.8 \\
Loss coefficient of accumulator valve $R_{A V}\left(\mathrm{GPa} \cdot \mathrm{s} / \mathrm{m}^{3}\right)$ & 1.5 \\
\hline
\end{tabular}

density functions of the road surface model, respectively. They can be described as in Ref. [24]:

$$
\begin{array}{r}
S_{d}(n)=S_{d}\left(n_{0}\right)\left(\frac{n}{n_{0}}\right)^{-\kappa}, \\
S_{r}(n)=\left[\frac{2 n_{0}^{\kappa} S_{d}\left(n_{0}\right)(\pi B / n)^{\kappa / 2}}{\boldsymbol{G a}(\kappa / 2)}\right] \boldsymbol{B} \boldsymbol{e}(2 \pi B n),
\end{array}
$$

where $n=f / v$ is the spatial frequency, $n_{0}$ is the reference spatial frequency $\left(n_{0}=0.1\right), S_{d}\left(n_{0}\right)$ is the spatial PSD at the reference spatial frequency, $\mathrm{K}=2$ is the undulation exponent, and $B$ is the wheel track. The symbols $\boldsymbol{G} \boldsymbol{a}$ and $\boldsymbol{B e}$ are defined as Gamma and Bessel functions, respectively. The diagonal elements of $S_{\text {Xout }}(f)$ represent the direct spectral densities of the output variable $X_{\text {out }}$.

\section{Numerical Results and Discussions}

The performance comparisons of mining vehicles with the CS and HIS systems are presented in this section. For the mining vehicle with the CS system, the stiffness of mechanical suspensions $k_{p s j}(j=f r, f l, r r, r l)$ is designed to be stiff to increase the pitch mode stiffness. However, the stiff suspension can cause poor ride comfort. Thus, The CS system cannot well coordinate bounce with the pitch vibrations of the mining vehicles. For the mining vehicle with the HIS system, the stiffness of the mechanical suspensions $k_{b s j}$ can be designed to be soft, which is smaller than $k_{p s j}$, and the hydraulic subsystem can provide additional pitch mode stiffness. For the simplification, the vehicle model with CS (equivalent stiffness $k_{p s j}$ ) and HIS are defined as VCS-EP and VHIS, respectively. The dynamic performance of the roll motion is not the research emphasis in this study owing to the low velocity of the mining vehicles not exceeding $50 \mathrm{~km} / \mathrm{h}$. The basic physical parameters of a seven-DOF vehicle model are given in Table 1. Table 2 lists the hydraulic parameters of the HIS system.

\subsection{Dynamic Performance Evaluation}

The identified natural frequencies, damping ratios, and corresponding mode shapes of VCS-EP and VHIS are presented in Table 5. As shown in Table 5, vehicle bodywheel motion-modes referring to the relative motion between body and wheels can be classified into seven modes in terms of the modal shapes. The normalized factor of the state vectors is used to distinguish seven modes. The motion corresponding to the maximum absolute value of the normalized factor in each modal shape represents one mode of the body-wheel motions. The values in the black bold font reveal that the first three mode shapes are called the body-dominated motionmodes, namely roll (1st), bounce (2nd), and pitch (3rd) motions of the sprung mass, and the following four mode shapes are called the wheel-dominated motion-modes, namely, the pitch (4th), bounce (5th), roll (6th), and warp (7th) motions of the unsprung mass. For the VCS-EP, the equivalent stiffness $k_{p s j}(j=f l, f r, r l, r r)$ is $265 \mathrm{~N} / \mathrm{mm}$. For
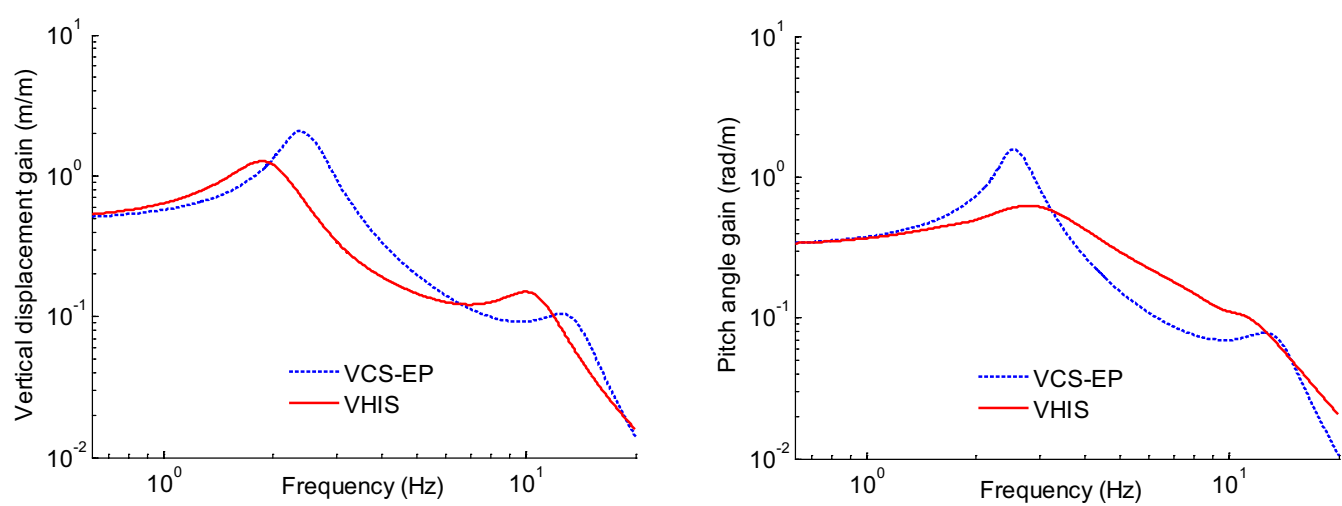

Figure 5 CG's bounce displacement and pitch angle under font wheel input 

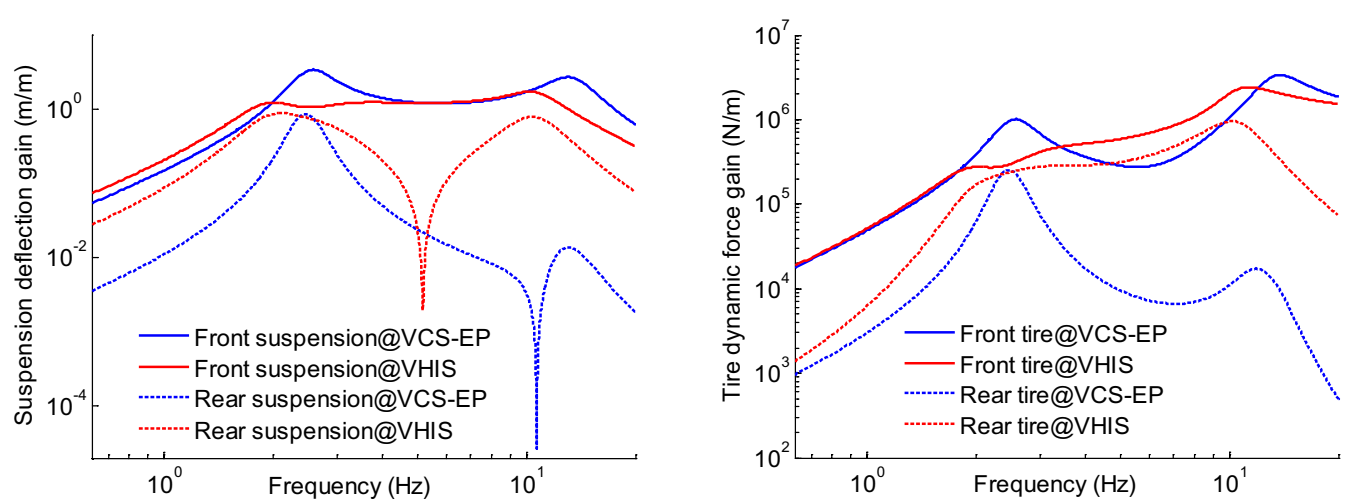

Figure 6 Suspension deflections and tire dynamic forces under front wheel input
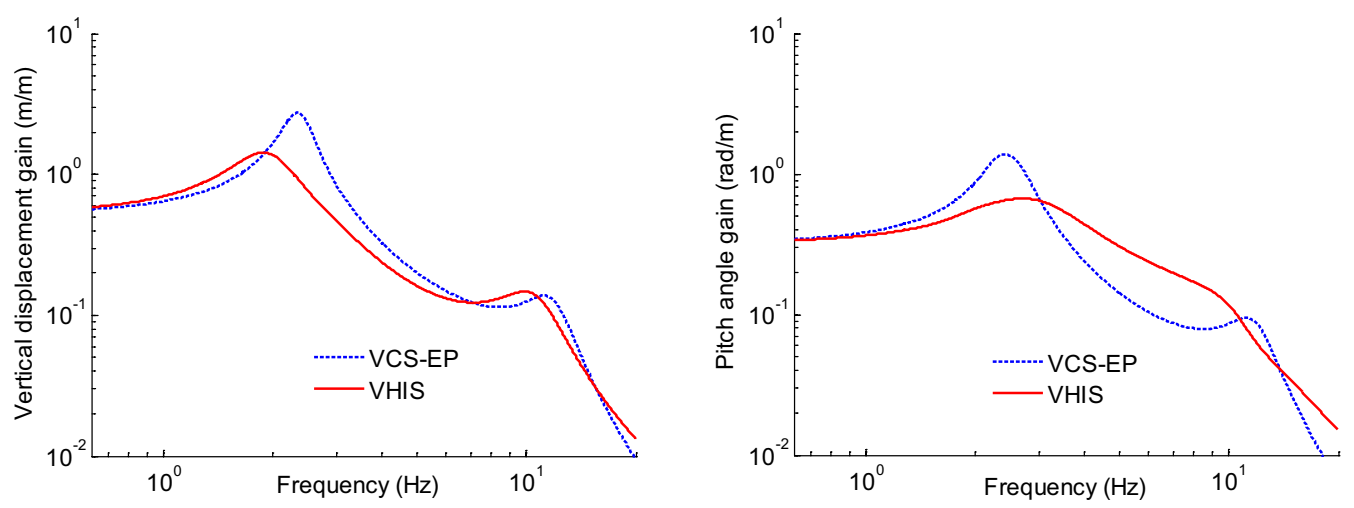

Figure 7 CG's bounce displacement and pitch angle under rear wheel input
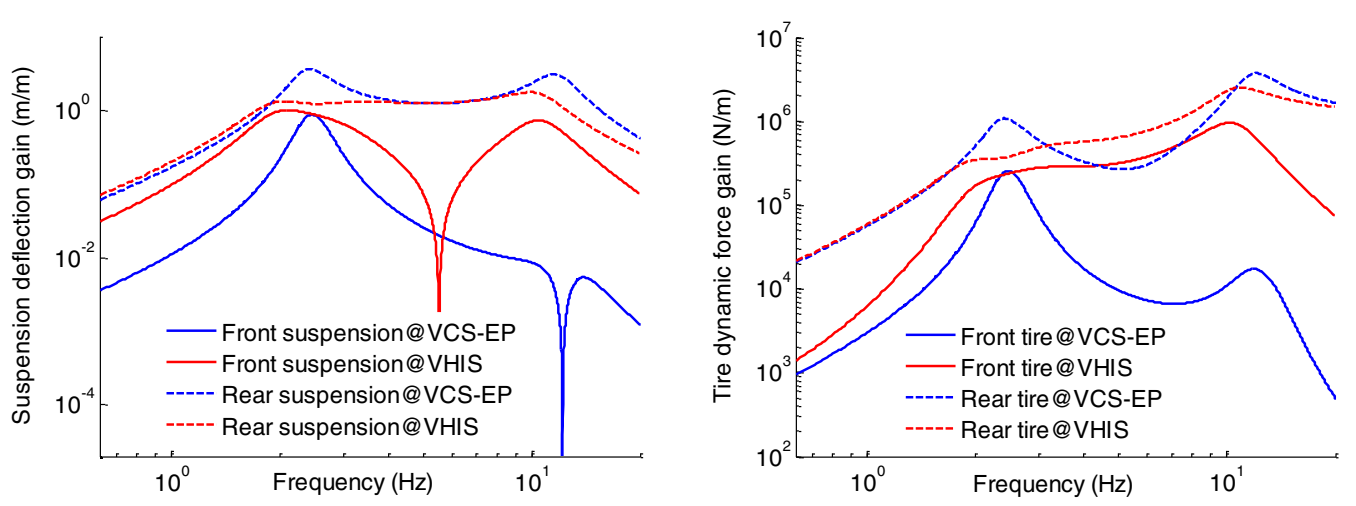

Figure 8 Suspension deflections and tire dynamic forces under rear wheel input

the VHIS, the equivalent stiffness $k_{b s i}(i=f l, f r)$ and $k_{b s j}$ $(j=r l, r r)$ are $129.2 \mathrm{~N} / \mathrm{mm}$ and $167.4 \mathrm{~N} / \mathrm{mm}$, respectively. In Table 5 , the HIS system can effectively increase the pitch mode (the 3rd mode) frequency (from $2.539 \mathrm{~Hz} /$ VCS-EP to $2.747 \mathrm{~Hz} / \mathrm{VHIS}$ ) of the sprung mass vibration, and reduce the bounce mode (the 2nd mode) frequency (from $2.351 \mathrm{~Hz} / \mathrm{VCS}-\mathrm{EP}$ to $1.906 \mathrm{~Hz} / \mathrm{VHIS}$ ). The result illustrates that the proposed HIS system can provide a higher pitch mode stiffness owing to the large fluids rapidly flowing into the accumulator in the pitch mode, and the lower bounce mode stiffness owing to the large fluids freely flowing in the hydraulic circuits. Owing to the 
soft mechanical suspensions, the body roll mode (the 1st mode) frequencies $(1.286 \mathrm{~Hz} / \mathrm{VHIS})$ of the VHIS are lower than that of the VCS-EP. It is also observed that the proposed HIS system is unable to provide the extra roll mode stiffness.

Additionally, the HIS system can reduce the natural frequencies of the wheel-dominated motion-modes, involving the wheel pitch mode (the 4th mode in VHIS, 9.158 $\mathrm{Hz}$ ), wheel bounce mode (the 5th mode in VHIS, 10.419 $\mathrm{Hz}$ ), wheel roll mode (the 6th mode in VHIS, $11.226 \mathrm{~Hz}$ ), and wheel warp mode (the 7th mode in VHIS, 12.530 $\mathrm{Hz}$ ). In the previous study [23], it can be seen that the lower warp mode stiffness can improve the ground holding performance. From the comparisons of the mode shapes, the HIS system can decouple the body bounce and pitch modes, and intensify the coupling of wheel bounce and pitch modes, which is beneficial to balance the distribution of the front and rear tire forces. Furthermore, it is observed that the HIS system can increase the damping ratios corresponding to both the body modes (roll, bounce, and pitch) and wheel modes (bounce and pitch) of the integrated system, and the high damping can eliminate transient oscillations.

According to the FRF matrix $\boldsymbol{H}_{\text {dis }}$ defined by Eqs. (27) and (28), the bilogarithmic graphs of the frequency response of CG's bounce displacement, pitch angle, suspension deflections, and tire dynamic forces for the VCS-EP and VHIS under the front wheel input $\left(\boldsymbol{w}=\left[\begin{array}{ll}0 & 0\end{array}\right.\right.$

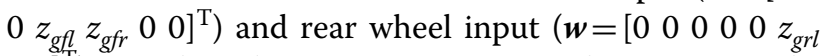
$\left.z_{g r r}\right]^{\mathrm{T}}$ ) are plotted in Figures 5, 6, 7 and 8, respectively. It shows that the HIS system reduces the peak vibration frequency for the bounce mode and increases the peak vibration frequency for the pitch mode. Under the front and rear wheel inputs, the peak values of the frequency response for the VHIS in the bounce and pitch modes are smaller than those of the VCS-EP. For the bounce mode, the transmissibility of the VHIS is suppressed if the frequency is less than $8 \mathrm{~Hz}$. For the pitch mode, the frequency response of the VHIS located before and after the natural vibration frequency is slightly suppressed and enlarged, respectively.

As shown in Figures 6 and 8, the peak values of the front suspension deflections and front tire dynamic forces are reduced by the HIS under the front wheel input. For the front suspension deflections of the VHIS, the frequency response lower than the natural vibration frequency is slightly enlarged, and that higher than the natural vibration frequency is unchanged; further, the HIS system reduces the transmissibility in high frequency ranges. For the front tire dynamic forces, the proposed HIS system maintains the same transmissibility located before the oscillation frequency, but slightly increases the transmissibility higher than the natural vibration frequency, and the frequency response is reduced in higher frequency ranges. Moreover, the frequency responses of the rear suspension deflections and rear tire dynamic forces under the left wheel input for the VHIS are much larger than those of the VCS-EP. This phenomenon illustrates that a mining vehicle with the HIS system transmits a greater degree of motion to the opposite wheels under the corresponding mode vibration. As shown, the frequency responses of the VHIS exhibit much broader peak ranges than the VCS-EP owing to the interconnection mechanism. For the VHIS, the additional excitation applied to the front or rear wheels can be transmitted much more efficiently to the opposite wheel through the hydraulic system. This result is helpful to balance the dynamic forces between the front and rear wheels, thus enhancing the ground holding performance.

The frequency responses of the bounce and pitch modes motion-energy for the VCS-EP and VHIS under the front and rear wheel inputs are shown in Figure 9. The transmissibility of the bounce mode motion-energy is reduced by the HIS system, but the frequency response
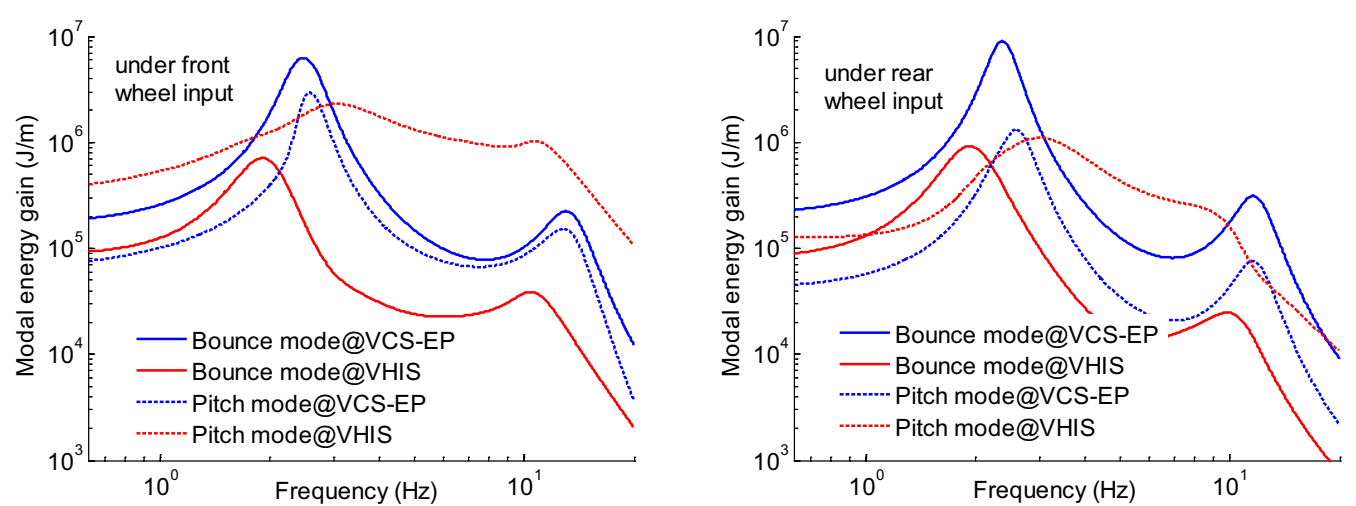

Figure 9 Motion-energy transmissibility under front wheel front and rear wheel inputs 


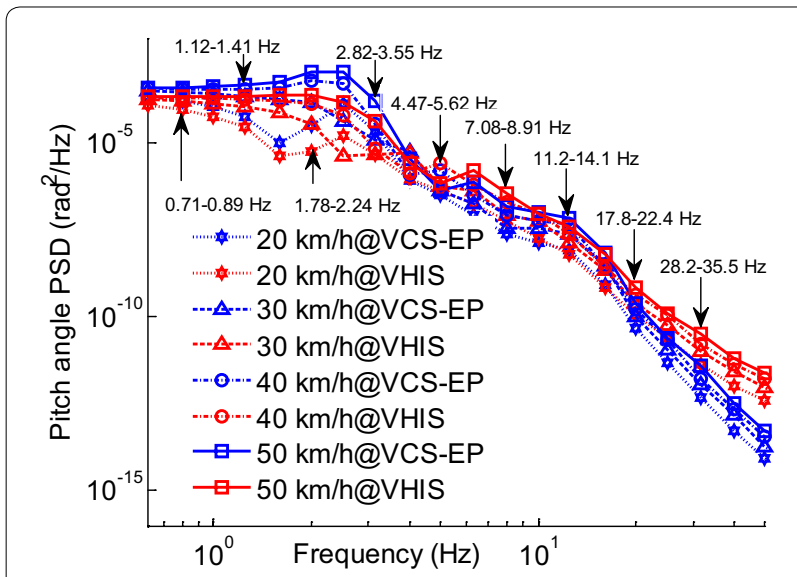

Figure 10 Pitch angle response in one-third octave bands under rough road

of the pitch mode motion-energy is enlarged. Based on the mode motion-energy definition, the complex eigenvalues exhibit a significant effect on the mode motionenergy. From Table 5, the modulus of the bounce mode eigenvalue for the VHIS is smaller than that of the VCS-EP, but the value of the pitch mode eigenvalue is larger than that of the VCS-EP. This is clearly a reflection that the vehicle with a softer bounce mode stiffness has a lower bounce mode motion-energy, and that with a stiffer pitch mode stiffness has a higher pitch mode motion-energy. Thus, these results show that the proposed HIS system can reduce the bounce mode stiffness and increase the pitch mode stiffness.

To evaluate the vehicle dynamic performances, the road surface spectrums including two types of the random road model (medium-smooth and rough roads) are applied in the simulation. Based on the road model description in Ref. [28], $S_{d}\left(n_{0}\right)=0.64 \times 10^{6}$ and $10.64 \times 10^{6}$ are used to describe these two average road types. The comparisons of PSD responses for the VCS-EP and VHIS with different speeds $(20,30,40$, and $50 \mathrm{~km} / \mathrm{h})$ are presented to investigate the suspension properties. The frequency-weighted root mean square (RMS) bounce, pitch angular and roll angular accelerations of the vehicle body are used to quantify the ride comfort
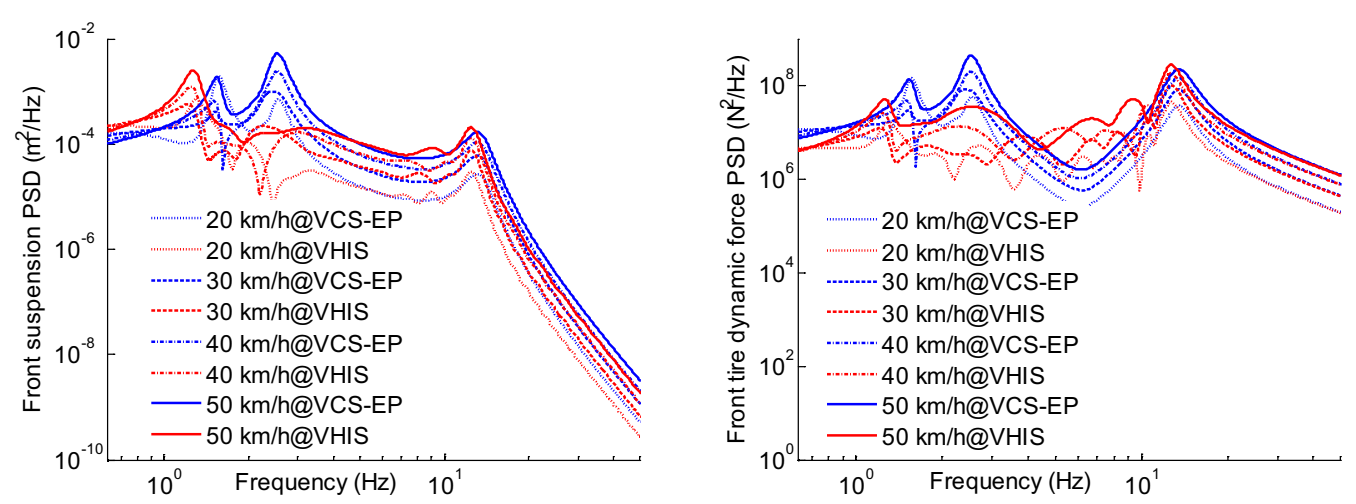

Figure 11 Front suspension deflection and tire dynamic force under rough road
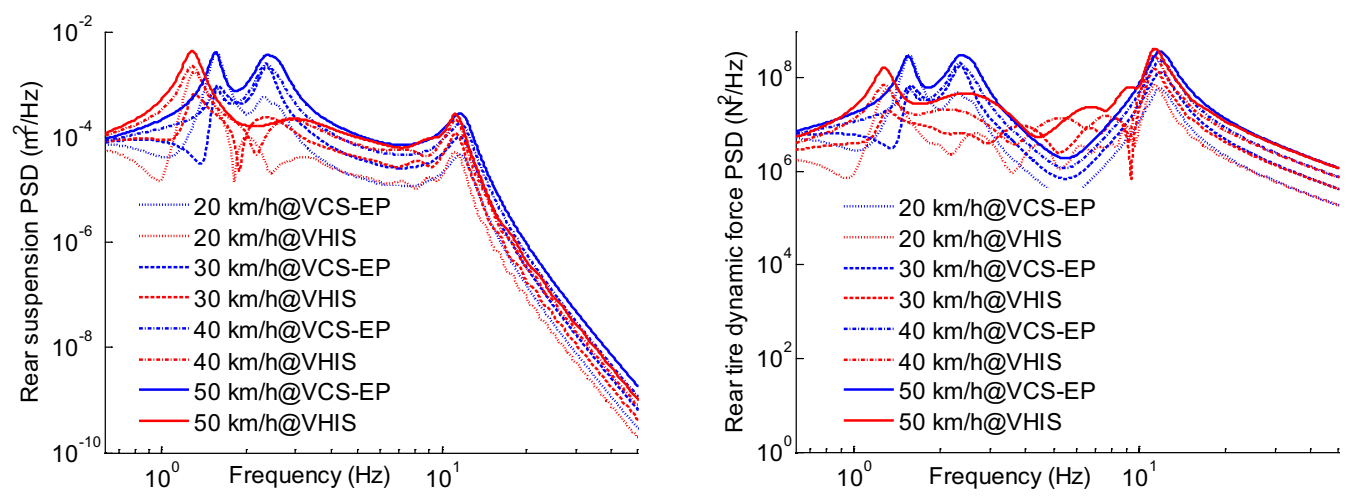

Figure 12 Rear suspension deflection and tire dynamic force under rough road 
Table 3 RMS acceleration comparisons

\begin{tabular}{|c|c|c|c|c|c|c|c|c|c|c|c|c|}
\hline \multirow[t]{2}{*}{ Speed } & \multicolumn{4}{|c|}{ Vertical acceleration $\left(\mathrm{m} / \mathrm{s}^{2}\right)$} & \multicolumn{4}{|c|}{ Pitch angular acceleration ( $\mathrm{rad} / \mathrm{s}^{2}$ ) } & \multicolumn{4}{|c|}{ Roll angular acceleration $\left(\mathrm{rad} / \mathrm{s}^{2}\right.$ ) } \\
\hline & 20 & 30 & 40 & 50 & 20 & 30 & 40 & 50 & 20 & 30 & 40 & 50 \\
\hline \multicolumn{13}{|c|}{ Medium-smooth road } \\
\hline VCS-EP & 0.783 & 1.515 & 1.621 & 1.610 & 0.399 & 0.482 & 0.871 & 1.245 & 1.712 & 1.087 & 1.214 & 2.00 \\
\hline VHIS & 0.614 & 0.886 & 1.102 & 1.260 & 0.274 & 0.410 & 0.612 & 0.833 & 1.101 & 0.873 & 1.454 & 2.100 \\
\hline Percentage & $21.58 \%$ & $41.52 \%$ & $32.02 \%$ & $21.74 \%$ & $31.33 \%$ & $14.94 \%$ & $29.74 \%$ & $33.09 \%$ & 35.69 & $19.69 \%$ & $19.77 \% \uparrow$ & $5 \% \uparrow$ \\
\hline \multicolumn{13}{|l|}{ Rough road } \\
\hline VCS-EP & 3.091 & 6.061 & 6.483 & 6.439 & 1.557 & 1.846 & 3.484 & 4.980 & 6.848 & 4.347 & 4.855 & 6.016 \\
\hline VHIS & 2.458 & 3.542 & 4.200 & 4.842 & 1.099 & 1.641 & 2.447 & 3.321 & 4.430 & 3.494 & 5.219 & 6.252 \\
\hline Percentage & $20.49 \%$ & $41.56 \%$ & $35.22 \%$ & $24.80 \%$ & $29.42 \%$ & $20.50 \%$ & $29.76 \%$ & $33.31 \%$ & $35.31 \%$ & $19.62 \%$ & $7.49 \% \uparrow$ & $3.92 \% \uparrow$ \\
\hline
\end{tabular}

according to ISO-2631-1 [29], and they can be written as follows:

$$
a_{i}=\sqrt{\chi_{i}^{2}(f) \boldsymbol{S}_{\text {Xoutjj }}(f)}, \quad i=b, p, r \quad j j=11,22,33,
$$

where $X_{b}, X_{p}$, and $X_{r}$ are the weighting factors of the bounce, pitch, and roll modes, respectively; $\boldsymbol{S}_{\text {Xout 11 }}, \boldsymbol{S}_{\text {Xout } 22}$, and $\boldsymbol{S}_{\text {Xout } 33}$ are the PSD responses of the CG's vertical, pitch, and roll angular accelerations, respectively.

Figure 10 shows the pitch angular response in one-third octave bands for the VCS-EP and VHIS under the rough road. The HIS system can effectively reduce the pitch angle of the vehicle body in a low frequency range, and maintain the pitch angle response in the range of 11.214.1 Hz, but slightly increase the frequency response in high frequency ranges. This is owing to the higher pitch stiffness provided by the HIS system. The PSD responses of the suspension deflections and tire dynamic forces for the VCS-EP and VHIS under the rough road are shown in Figures 11 and 12. The frequency responses of the suspension deflections and tire dynamic forces are reduced by the HIS system in low frequency ranges, and those in high frequency ranges are unchanged. The first natural vibration frequency of the PSD response is reduced for the VHIS with different speeds from 20 to $50 \mathrm{~km} / \mathrm{h}$ owing to the lower roll stiffness. The PSD response peak values of the suspension deflections and tire dynamic forces for the VHIS are evidently smaller than those of the VCSEP. These results show that the HIS system can reduce the suspension deflections and tire dynamic forces to improve the holding ground performance, and decrease the bounce and pitch angular accelerations to improve the ride comfort for the mining vehicle.

The RMS acceleration comparisons of the bounce, pitch, and roll modes between the VHIS and VCS are shown in Table 3. It shows that the proposed HIS can suppress the bounce and pitch motions of the vehicle body. The RMS vertical accelerations under the medium-smooth road with different speeds have been decreased from 0.783 to $0.614 \mathrm{~m} / \mathrm{s}^{2}, 1.515$ to $0.886 \mathrm{~m} /$ $\mathrm{s}^{2}, 1.621$ to $1.102 \mathrm{~m} / \mathrm{s}^{2}$, and 1.610 to $1.260 \mathrm{~m} / \mathrm{s}^{2}$. The HIS system has significantly reduced the RMS vertical accelerations under the rough road by $20.49 \%, 41.56 \%$, $35.22 \%$, and $24.80 \%$, respectively. Meanwhile, the RMS pitch angular accelerations under medium-smooth and rough roads have been decreased by no less than $14.5 \%$ and $20 \%$, respectively. The RMS roll angular accelerations at low speeds $(20$ and $30 \mathrm{~km} / \mathrm{h}$ ) are almost reduced by $35 \%$ and $20 \%$, respectively. And the HIS system has slightly increased the RMS roll angular accelerations at high speeds $(40$ and $50 \mathrm{~km} / \mathrm{h}$ ) within an accepted variance. These results demonstrate that the proposed HIS system can improve the mining vehicle ride comfort.

\subsection{Parametric Sensitivity Analysis}

To further illustrate the decoupled advantages of the proposed HIS, the parametric sensitivity analysis of the damper valves is presented in this section, including the cylinder valve $(\mathrm{CV})$, hydraulic circuit valve $(\mathrm{HCV})$, and accumulator valve (AV). These parameters are employed to analyze their effects on the vibration transmissibility of the vehicle body bounce, pitch, and roll modes. Figure 12 shows the frequency responses of the CG's bounce displacement, pitch angle, and roll angle with different loss coefficients for the VHIS under the front-left wheel input $\boldsymbol{w}=\left[\begin{array}{lllllll}0 & 0 & 0 & z_{g f l} & 0 & 0 & 0\end{array}\right]^{\mathrm{T}}$. The front-left wheel input can easily induce the body bounce, pitch, and roll modes. From Figure 13(a), the $\mathrm{CV}$ provides the higher roll mode damping than the bounce mode damping, and has little influence on the pitch mode damping. Further, it is observed that the higher CV loss coefficient leads to a stiffer roll mode. From Figure 13(b), the HCV appears to generate a 

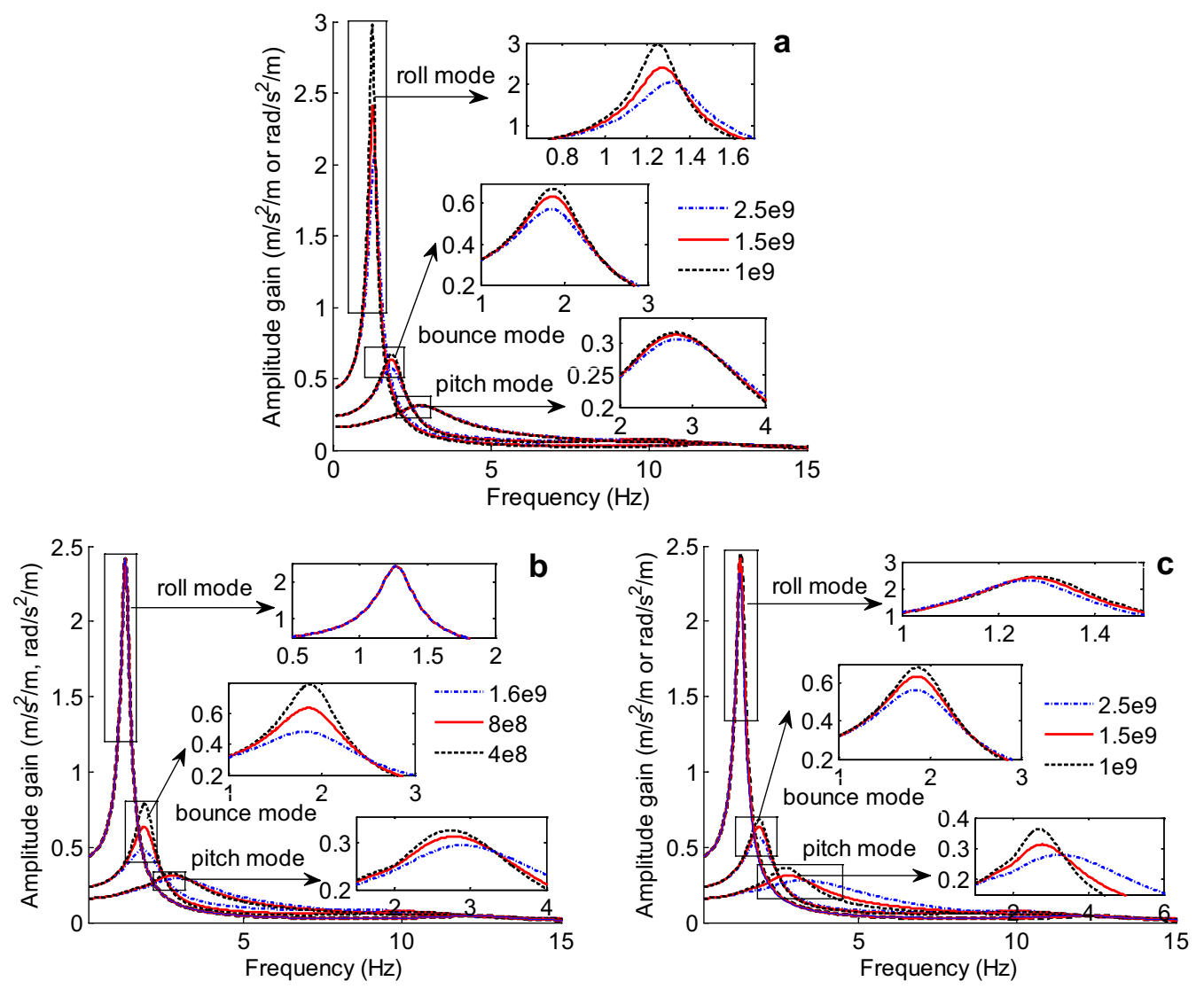

Figure 13 Vibration transmissibility with different loss coefficients: (a) CV, (b) HCV, (c) AV

Table 4 Comparisons of natural frequency and damping ratio

\begin{tabular}{|c|c|c|c|c|c|c|c|c|c|}
\hline & \multicolumn{3}{|c|}{$R_{C V}\left(\mathrm{GPa} \cdot \mathrm{s} / \mathrm{m}^{3}\right)$} & \multicolumn{3}{|c|}{$R_{H C V}\left(\mathrm{GPa} \cdot \mathrm{s} / \mathrm{m}^{3}\right)$} & \multicolumn{3}{|c|}{$R_{A V}\left(\mathrm{GPa} \cdot \mathrm{s} / \mathrm{m}^{3}\right)$} \\
\hline & 1 & 1.5 & 2.5 & 0.4 & 0.8 & 1.6 & 0.1 & 1.5 & 2.5 \\
\hline \multicolumn{10}{|c|}{ Roll mode } \\
\hline$f_{d}(\mathrm{~Hz})$ & 1.264 & 1.286 & 1.329 & 1.286 & 1.286 & 1.286 & 1.296 & 1.286 & 1.271 \\
\hline$\xi$ & 0.077 & 0.097 & 0.117 & 0.097 & 0.097 & 0.097 & 0.097 & 0.097 & 0.099 \\
\hline \multicolumn{10}{|c|}{ Bounce mode } \\
\hline$f_{d}(\mathrm{~Hz})$ & 1.909 & 1.907 & 1.902 & 1.9125 & 1.907 & 1.890 & 1.909 & 1.907 & 1.901 \\
\hline$\xi$ & 0.190 & 0.205 & 0.235 & 0.159 & 0.205 & 0.300 & 0.190 & 0.205 & 0.237 \\
\hline \multicolumn{10}{|c|}{ Pitch mode } \\
\hline$f_{d}(\mathrm{~Hz})$ & 2.741 & 2.747 & 2.761 & 2.732 & 2.747 & 2.796 & 2.714 & 2.747 & 3.083 \\
\hline$\xi$ & 0.348 & 0.358 & 0.378 & 0.327 & 0.358 & 0.423 & 0.267 & 0.358 & 0.551 \\
\hline
\end{tabular}

significant effect on the bounce mode characteristic. However, it is nearly unrelated to the dynamic characteristics of the roll mode. From Figure 13(c), the AV is primarily designed to control the pitch mode property. The comparisons of natural frequency and damping ratio with the $\mathrm{CV}, \mathrm{HCV}$ and $\mathrm{AV}$ loss coefficients are presented in Table 4. These results are in accord with the above phenomena, and prove that the proposed HIS system can decouple the vehicle body modes by adjusting the corresponding loss coefficient, which is the irreconcilable contradiction for the CS system. 
Table 5 The comparisons of natural frequency and mode shape

\begin{tabular}{|c|c|c|c|c|c|c|c|}
\hline & \multicolumn{7}{|l|}{ Mode shape } \\
\hline & 1st & 2nd & $3 r d$ & 4th & 5 th & 6th & 7th \\
\hline Description & $\begin{array}{l}\text { Body-dominated } \\
\text { roll mode }\end{array}$ & $\begin{array}{l}\text { Body-dominated } \\
\text { bounce mode }\end{array}$ & $\begin{array}{l}\text { Body-dominated } \\
\text { pitch mode }\end{array}$ & $\begin{array}{l}\text { Wheel-dominated } \\
\text { pitch mode }\end{array}$ & $\begin{array}{l}\text { Wheel-domi- } \\
\text { nated bounce } \\
\text { mode }\end{array}$ & $\begin{array}{l}\text { Wheel-domi- } \\
\text { nated roll mode }\end{array}$ & $\begin{array}{l}\text { Wheel-dominated } \\
\text { warp mode }\end{array}$ \\
\hline \multicolumn{8}{|l|}{ VCS-EP } \\
\hline s & $-0.72+9.83 i$ & $-1.62+14.77 i$ & $-1.90+15.95 i$ & $-10.27+73.51 i$ & $-13.17+83.50 i$ & $-9.64+73.69 i$ & $-12.32+83.83 i$ \\
\hline$f_{d}(\mathrm{~Hz})$ & 1.564 & 2.351 & 2.539 & 11.699 & 13.286 & 11.728 & 13.342 \\
\hline$\xi$ & 0.073 & 0.109 & 0.118 & 0.138 & 0.156 & 0.129 & 0.145 \\
\hline$z_{s}$ & 0 & 1 & $-0.525-0.005 i$ & $-0.029-0.030 \mathrm{i}$ & $-0.023-0.026 i$ & 0 & 0 \\
\hline$\theta$ & 0 & $0.240+0.002 i$ & 1 & $-0.020-0.021 i$ & $0.017+0.020 i$ & 0 & 0 \\
\hline$\varphi$ & 1 & 0 & 0 & 0 & 0 & $-0.037-0.036 i$ & $0.019+0.022 i$ \\
\hline$z_{\text {ufl }}$ & $0.059+0.009 i$ & $0.114+0.026 i$ & $-0.406-0.104 i$ & $-0.003+0.007 i$ & 1 & $0.000-0.021 i$ & -1 \\
\hline$z_{u f r}$ & $-0.059-0.009 i$ & $0.114+0.026 i$ & $-0.406-0.104 i$ & $-0.003+0.007 i$ & 1 & $-0.000+0.021 \mathrm{i}$ & 1 \\
\hline$z_{u r l}$ & $0.086+0.013 i$ & $0.257+0.061 i$ & $0.184+0.046 i$ & 1 & $0.001-0.005 i$ & 1 & $0.001-0.014 i$ \\
\hline $\begin{array}{l}z_{\text {urr }} \\
\text { VHIS }\end{array}$ & $-0.086-0.013 i$ & $0.257+0.061 i$ & $0.184+0.046 i$ & 1 & $0.001-0.005 i$ & -1 & $-0.001+0.014 i$ \\
\hline s & $-0.81+8.08 i$ & $-2.40+12.00 i$ & $-6.79+17.18 i$ & $-32.02+57.63 i$ & $-11.97+65.45 i$ & $-6.01+70.55 i$ & $-7.99+78.71 i$ \\
\hline$f_{d}(\mathrm{~Hz})$ & 1.286 & 1.906 & 2.747 & 9.158 & 10.419 & 11.226 & 12.530 \\
\hline$\xi$ & 0.097 & 0.205 & 0.358 & 0.488 & 0.179 & 0.087 & 0.099 \\
\hline$z_{s}$ & 0 & 1 & $0.001-0.097 i$ & $-0.002-0.083 i$ & $0.030-0.087 i$ & 0 & 0 \\
\hline$\theta$ & 0 & $-0.013+0.036 i$ & 1 & $0.043+0.166 i$ & $-0.032-0.004 i$ & 0 & 0 \\
\hline$\varphi$ & 1 & 0 & 0 & 0 & 0 & $0.020+0.027 i$ & $0.007+0.017 i$ \\
\hline$z_{\text {uff }}$ & $0.034+0.008 i$ & $0.114+0.045 i$ & $-0.273-0.305 i$ & $-0.833-0.187 i$ & $0.945+0.263 i$ & $-0.004+0.009 i$ & -1 \\
\hline$z_{\text {ufr }}$ & $-0.034-0.008 i$ & $0.114+0.045 i$ & $-0.273-0.305 i$ & $-0.833-0.187 i$ & $0.945+0.263 i$ & $0.004-0.009 i$ & 1 \\
\hline$z_{\text {url }}$ & $0.061+0.011 i$ & $0.117+0.060 i$ & $0.314+0.272 i$ & 1 & 1 & -1 & $0.004-0.006 i$ \\
\hline$z_{\text {urr }}$ & $-0.061-0.011 i$ & $0.117+0.60 i$ & $0.314+0.272 i$ & 1 & 1 & 1 & $-0.004+0.006 i$ \\
\hline
\end{tabular}

\section{Conclusions and Future Work}

A new HIS system to was proposed herein to improve the bounce and pitch motion performances for a mining vehicle. The integrated dynamic equations of the mechanical-hydraulic coupled system were derived using the impedance transfer matrix and free-body diagram methods. Based on the derived equations, the modal analysis method was employed to obtain the free vibration transmissibilities and force vibration responses under different road excitations. The dynamic characteristic comparisons between the VCS-EP and VHIS were presented to verify the prominent advantages of the HIS system. These results illustrated that the proposed HIS system could decouple the bounce and pitch modes, and intensify the coupling of wheel bounce and pitch modes to balance the distribution of tire dynamic forces. Furthermore, the HIS system could provide additional pitch stiffness to control the vehicle pitch motion, reduce the bounce stiffness to improve ride comfort, and increase the corresponding mode damping to eliminate transient oscillations in the lower frequency range.
Herein, the most obvious limitation is that the HIS system could not provide adequate roll stiffness to control the roll mode. Another limitation was that the modal analysis method was linear in this study. However, the suspension system and tire assembly often exhibit many nonlinearities for practical applications. Thus, the performances of the bounce, pitch, and roll modes would be considered for the passive HIS system, and the extension of the proposed methodology to a full nonlinear vehicle model must be explored in the future. Additionally, the semi-active or active control strategy for the HIS system must be considered as future works to improve the ride comfort and handling performance of the mining vehicle based on control theories.

\section{Authors' Contributions}

$J Z$ and YD was in charge of the whole trial; JZ wrote the manuscript; NZ, BZ, $\mathrm{HQ}$ and $\mathrm{MZ}$ assisted with sampling and laboratory analyses. All authors read and approved the final manuscript.

\section{Author Details}

${ }^{1}$ State Key Laboratory of Advanced Design and Manufacturing for Vehicle Body, College of Mechanical and Vehicle Engineering, Hunan University, 
Changsha 410082, China. ${ }^{2}$ Automotive Research Institute, Hefei University of Technology, Hefei 230009, China. ${ }^{3}$ School of Automotive and Transportation Engineering, Hefei University of Technology, Hefei 230009, China.

\section{Authors' Information}

Jie Zhang, born in 1987, is currently a PhD candidate at State Key Laboratory of Advanced Design and Manufacture for Vehicle Body, Hunan University, China. He received his bachelor degree from Yangtze University, China, in 2011. His research interests include control, modeling, and estimation of driver-vehicle system dynamics.

Yuanwang Deng, born in 1968, is currently an associate professor at College of Mechanical and Vehicle Engineering, Hunan University, China. He received his PhD degree from Huazhong University of Science and Technology, China, in 2002. His research interests include heat transfer theory, engine electronic control technology and mechanical system dynamics.

Nong Zhang, born in 1959, is currently a professor at Automotive Research Institute, Hefei University of Technology, China. He received his PhD degree from University of Tokyo, Japan, in 1989. His current research interests include dynamics and control of automotive systems.

Bangji Zhang, born in 1967, is currently a professor at State Key Laboratory of Advanced Manufacture and Design for Vehicle Body, Hunan University, China.

Hengmin Qi, born in 1990, is currently a PhD candidate at State Key Laboratory of Advanced Design and Manufacture for Vehicle Body, Hunan University, China. He received his bachelor's degree from China University of Petroleum China, in 2012. His research interests include design and analysis of hydraulically interconnected suspension system and vehicle system dynamics.

Minyi Zheng, born in 1983, is currently a lecturer at School of Automotive and Transportation Engineering, Hefei University of Technology, China. He received his Ph.D. from Hunan University, China, in 2016 His research interests include vehicle dynamics and parameter identification.

\section{Acknowledgements}

The authors sincerely thanks to Assistant Professor Fei Ding of Hunan University for his critical discussion and reading during manuscript preparation.

\section{Competing Interests}

The authors declare no competing financial interests.

\section{Funding}

Supported by National Natural Science Foundation of China (Grant Nos. 51805155, 51675152), Foundation for Innovative Research Groups of National Natural Science Foundation of China (Grant No. 51621004), and Open Fund in the State Key Laboratory of Advanced Design and Manufacture for Vehicle Body (Grant No. 71575005).

\section{Appendix}

Table 5 shows the comparisons of natural frequency and mode shape between the VCS-EP and VHIS.

\section{Publisher's Note}

Springer Nature remains neutral with regard to jurisdictional claims in published maps and institutional affiliations.

\section{Received: 27 March 2017 Accepted: 8 January 2019}

Published online: 29 January 2019

\section{References}

[1] TEger, J Stevenson, P É Boileau, et al. Predictions of health risks associated with the operation of load-haul-dump mining vehicles: Part 1—Analysis of whole-body vibration exposure using ISO 2631-1 and ISO-2631-5 standards. International Journal of Industrial Ergonomics, 2008, 38(9-10): 726-738.
[2] D P Cao, X B Song, M Ahmadian. Editors' perspectives: road vehicle suspension design, dynamics, and control. Vehicle System Dynamics, 2001, 49(1-2): 3-28.

[3] M C Smith, F C Wang. Controller parameterization for disturbance response decoupling: application to vehicle active suspension control. IEEE Transactions on Control Systems Technology, 2002, 10(3): 393-407.

[4] HY Li, H H Liu, H J Gao, et al. Reliable fuzzy control for active suspension systems with actuator delay and fault. IEEE Transactions on Fuzzy Systems, 2012, 20(2): 342-357.

[5] SY Han, CH Zhang, G Y Tang. Approximation optimal vibration for networked nonlinear vehicle active suspension with actuator time delay. Asian Journal of Control, 2017, 19(3): 983-995.

[6] W C Sun, H H Pan, H J Gao. Filter-based adaptive vibration control for active vehicle suspensions with electrohydraulic actuators. IEEE Transactions on Vehicular Technology, 2016, 65(6): 4619-4626.

[7] S Bououden, M. Chadli, H R Karimi. A robust predictive control design for nonlinear active suspension systems. Asian Journal of Control, 2016, 18(1): 122-132.

[8] Y B Huang, J Na, X Wu, et al. Adaptive control of nonlinear uncertain active suspension systems with prescribed performance. ISA Transactions, 2015, 54(1): 145-155.

[9] X J Wu, B Zhou, G L Wen, et al. Intervention criterion and control research for active front steering with consideration of road adhesion. Vehicle System Dynamics, 2018, 56 (4): 553-578.

[10] J Wu, S Chen, B H Liu, et al. A human-machine-cooperative-driving controller based on AFS and DYC for vehicle dynamic stability. Energies, 2017, 10(11): 17-37.

[11] S Chen, L Li, J Chen. Fusion algorithm design based on adaptive SCKF and integral correction for side-slip angle observation. IEEE Transactions on Industrial Electronics, 2018, 65(7): 5754-5763.

[12] J Zhao, P K Wong, X B Ma, et al. Chassis integrated control for active suspension, active front steering and direct yaw moment systems using hierarchical strategy. Vehicle System Dynamics, 2017, 55(1): 72-103.

[13] A M C Odhams, D Ceon. An analysis of ride coupling in automobile suspensions. Proceedings of the Institution of Mechanical Engineers, Part D: Journal of Automobile Engineering, 2006, 220(8): 1041-1061.

[14] M C Smith, G W Walker. Interconnected vehicle suspension. Proceedings of the Institution of Mechanical Engineers, Part D: Journal of Automobile Engineering, 2005, 219(3): 295-307.

[15] Z X Li, LY Ju, H Jiang, et al. Experimental and simulation study on the vibration isolation and torsion elimination performances of interconnected air suspensions. Proceedings of the Institution of Mechanical Engineers, Part D: Journal of Automobile Engineering, 2016, 230(5): 679-691.

[16] N Mace. Analysis and synthesis of passive interconnected vehicle suspensions. Cambridge: University of Cambridge, 2004.

[17] D P Cao, S Rakheja, CY Su. Dynamic analyses of roll plane interconnected hydro-pneumatic suspension systems. International Journal of Vehicle Design, 2008, 47(1): 51-80.

[18] S Bhave. Effect of connecting the front and rear air suspensions of a vehicle on the transmissibility of road undulation inputs. Vehicle System Dynamics, 1992, 21(1): 225-245.

[19] Q L Yao, X J Zhang, K H GUO, et al. Study on a novel dual-mode interconnected suspension. International Journal of Vehicle Design, 2015, 68(1-3): 81-103.

[20] K H Guo, Y H Chen, Y Zhuang, et al. Modeling and Simulation Study of Hydro-pneumatic Interconnected Suspension System. Journal of Hunan University (Natural Sciences), 2011, 38(3): 29-33. (in Chinese)

[21] N Zhang, W A SMITH, J Jeyakumaran. Hydraulically interconnected vehicle suspension: Background and modeling. Vehicle System Dynamics, 2010, 48(1): 17-40.

[22] F Ding, N Zhang, J Liu, et al. Dynamics analysis and design methodology of roll-resistant hydraulically interconnected suspensions for tri-axle straight trucks. Journal of the Franklin Institute Engineering and Applied Mathematics, 2016, 353(17): 4620-4651.

[23] S Z Zhu, G Z Xu, A Tkachev, et al. Comparison of the road-holding abilities of a roll-plane hydraulically interconnected suspension system and an anti-roll bar system. Proceedings of the Institution of Mechanical Engineers, Part D: Journal of Automobile Engineering, 2016, 231(11): 1540-1557.

[24] B Zhou, Y Geng, XT Huang. Global sensitivity analysis of hydraulic system parameters to hydraulically interconnected suspension dynamic 
response. International Journal of Vehicle Noise and Vibration, 2015, 11(2): 185-197.

[25] R C Wang, Q Ye, ZY Sun, et al. A study of the hydraulically interconnected inerter-spring-damper suspension system. Mechanics Based Design of Structures and Machines, 2017, 45(4): 415-429.

[26] L Li, J Song, Z Han, et al. Hydraulic model and inverse model for electronic stability program online control system. Journal of Mechanical Engineering, 2008, 44(2): 139-144. (in Chinese)

[27] L F Wang, N Zhang, H P Du. Real-time identification of vehicle motionmodes using neural networks. Mechanical Systems and Signal Processing 2015, 50-51: 632-645.
[28] A Pazooki, D P Cao, S Rakheja. Ride dynamic evaluations and design optimization of a torsio-elastic off-road vehicle suspension. Vehicle System Dynamics, 2011, 49(9): 1455-1476.

[29] International Organization for Standardization. ISO2631-1 Mechanical vibration and shock-evaluation of human exposure to whole-body Vibration-part1: General requirements. State College: The Organization, 1997.
Submit your manuscript to a SpringerOpen ${ }^{\ominus}$ journal and benefit from:

- Convenient online submission

- Rigorous peer review

- Open access: articles freely available online

- High visibility within the field

- Retaining the copyright to your article

Submit your next manuscript at $\boldsymbol{\nabla}$ springeropen.com 